DOE/BC/96001224

Distribution Category UC-122

\title{
Two-Dimensional Network Simulation of Diffusion Driven \\ Coarsening of Foam Inside a Porous Medium
}

Topical Report

By

David Cohen

T. W. Patzek

C. J. Radke

April 1996

\author{
Prepared for \\ U.S. Department of Energy \\ Assistant Secretary for Fossil Energy
}

\author{
Thomas Reid, Project Manager \\ Bartlesville Project Office \\ P.O. Box 1398 \\ Bartlesville, OK 74005
}
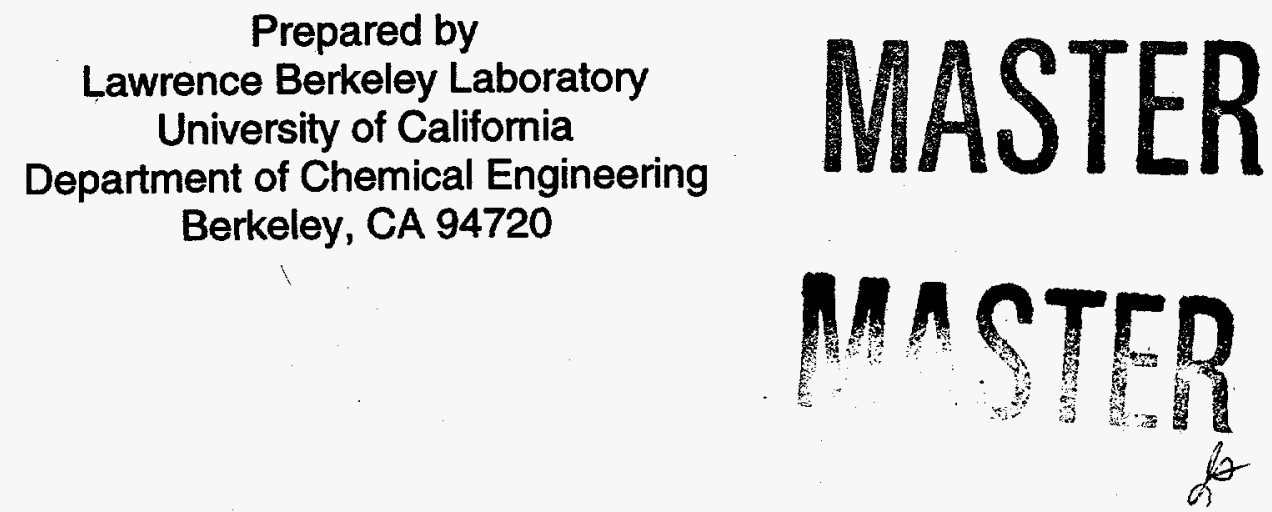


\section{DISCLAIMER}

Portions of this document may be illegible in electronic image products. Images are produced from the best available original document. 


\section{TABLE OF CONTENTS}

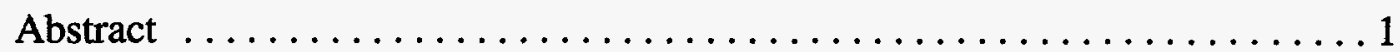

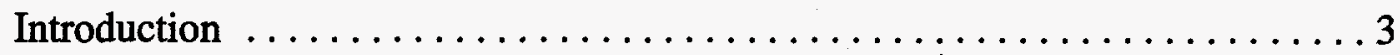

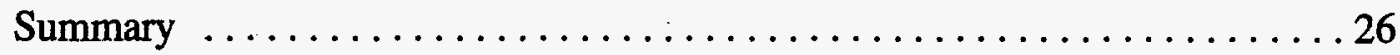

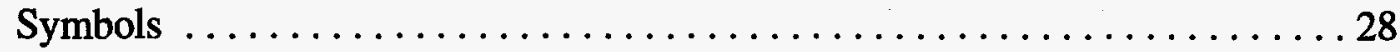

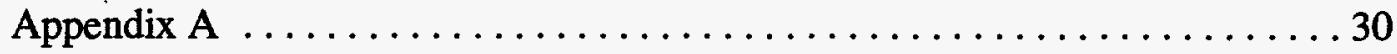

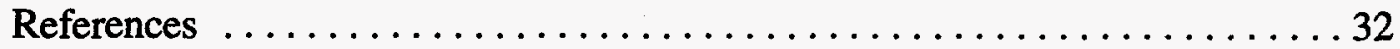

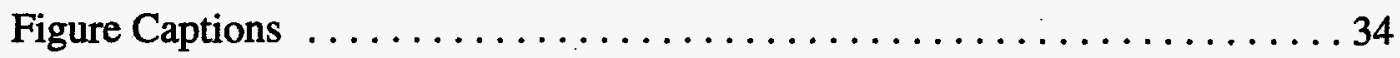

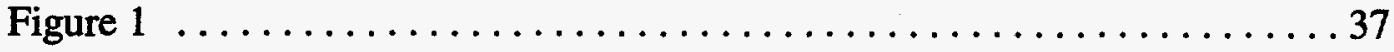

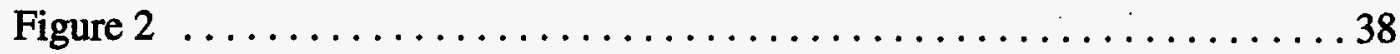

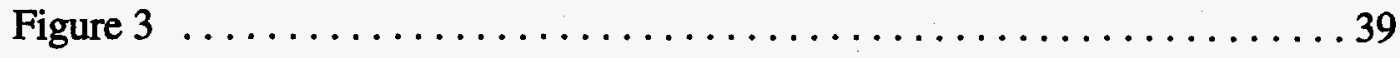

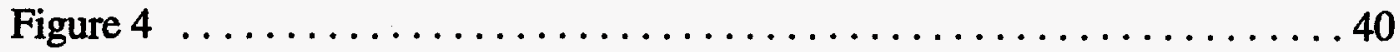

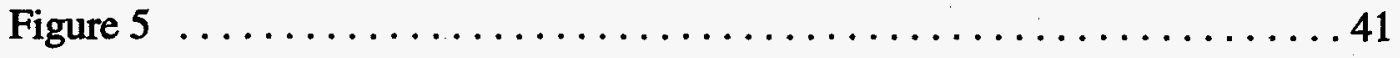

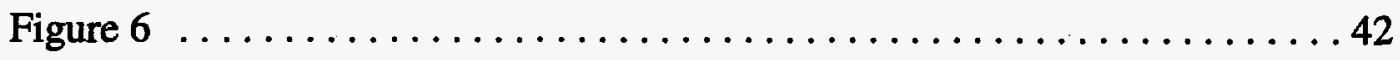

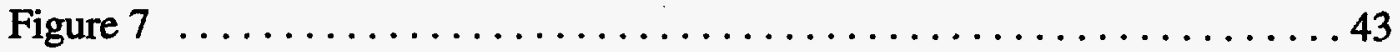

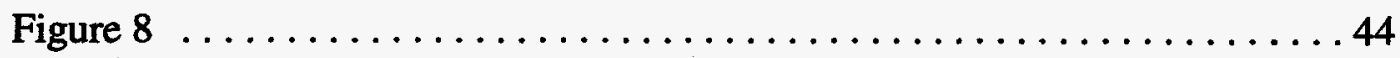

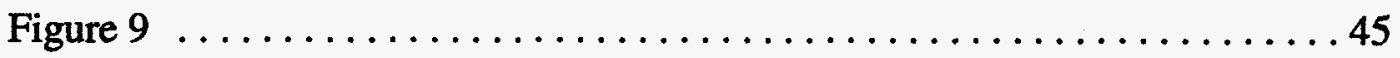

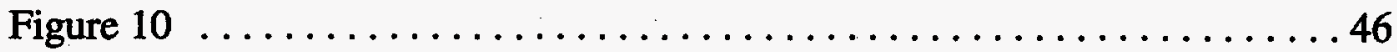

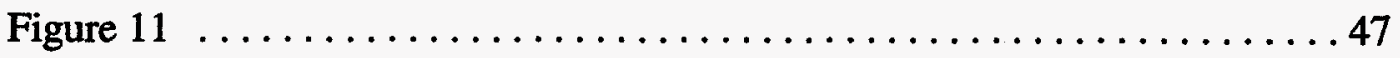

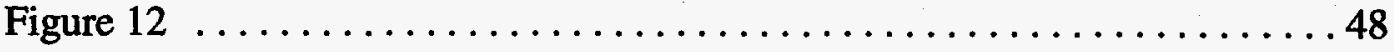

Figure $13 \ldots \ldots \ldots \ldots \ldots \ldots \ldots \ldots \ldots \ldots \ldots \ldots \ldots \ldots \ldots . \ldots \ldots$

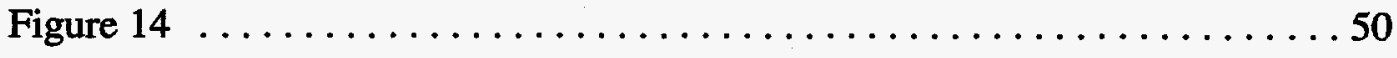

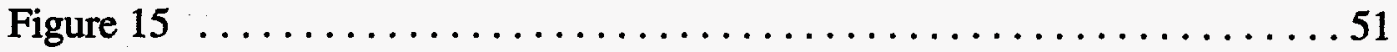

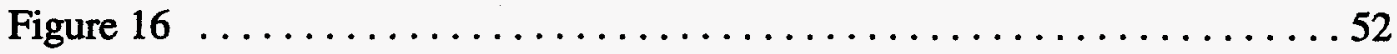

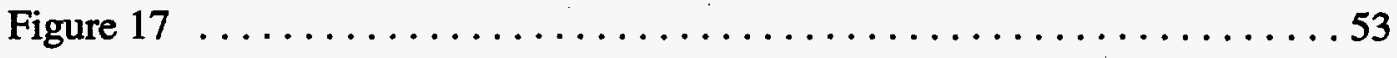

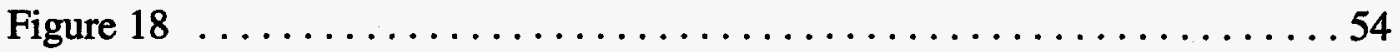

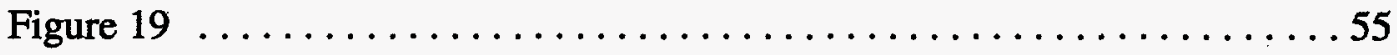

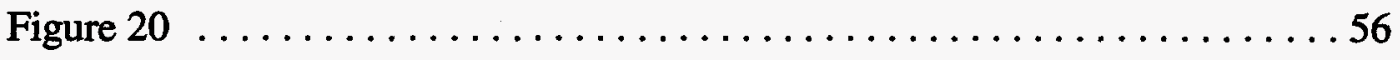

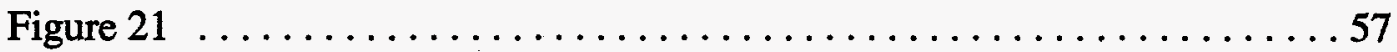




\title{
Two-Dimensional Network Simulation of Diffusion \\ Driven Coarsening of Foam Inside a Porous Medium \\ David Cohen,* T.W. Patzek, ${ }^{* *}$ and C.J. Radke*
}

Lawrence Berkeley National Laboratory, *Departments of Chemical Engineering, and

**Materials Science and Mineral Engineering, University of California-Berkeley, Berkeley, CA 94720

\begin{abstract}
In order to use foams in subsurface applications, it is necessary to understand their stability in porous media. Diffusion driven coarsening of a stationary or nonflowing foam in a porous medium results in changing gas pressures and a coarsening of the foam texture. A two-dimensional network simulation has been created that predicts the behavior of foam in a porous medium by physically specifying the locations of all the lamellae in the system and by solving the complete set of Young-Laplace and diffusion equations. An hourglass approximates the shape of the pores, and the pore walls are considered to be highly water wet. A singularity arises in the system of differential algebraic equations due to the curvature of the pore walls. This singularity is a signal that the system must undergo oscillations or sudden lamellar rearrangements before the diffusion process can continue. Newton-Raphson iteration is used along with Keller's method of arc-length continuation and a new jump resolution technique to locate and resolve bifurcations in the system of coupled lamellae.
\end{abstract}

Gas bubbles in pore throats are regions of encapsulated pressure. As gas is released from these bubbles during diffusion, the pressure of the bubbles in the pore bodies increases. When the pressure increase is scaled by the characteristic Young-Laplace pressure, the equilibrium time for the diffusion process is scaled by the ratio of the square of the characteristic length to the gas diffusivity and two dimensionless groups. One describes the ease with which gas can diffuse through a lamella, the second represents the amount of gas encapsulated within the pore throats initially. Given this scaling, the 
resulting plots of pressure versus time and normalized lamellae positions versus time are universal for all system sizes and characteristics. This is true as long as the initial lamella distribution is the same in each case.

Key Words: foam, porous media, diffusion, network simulation 


\section{INTRODUCTION}

Foam can significantly reduce the mobility of a gas flowing through a porous medium. Therefore, foam is a useful fluid for improving the recovery of oil in steam or $\mathrm{CO}_{2}$ flooding processes. 1,2 In addition, the low mobility makes foam a promising blocking fluid for underground gas storage, ${ }^{3}$ and for prevention of gas coning in production wells. ${ }^{4}$ To facilitate such applications, it is crucial to characterize the long term stability of foam in porous media.

Foam in a porous medium is stable below a limiting capillary pressure, which depends on several factors, including the surfactant properties, the gas velocity during

flow, and the permeability of the porous medium. ${ }^{5}$ When the limiting capillary pressure is exceeded, the liquid in the foam films cannot withstand the capillary suction which thins and eventually destabilizes the films. Our study looks at the coalescence of stationary foams under conditions where all lamellae are exposed to capillary suction pressures significantly below the limiting capillary pressure and are, therefore, unconditionally stable. Diffusion is the only mechanism which leads to foam coalescence, as gas diffuses across lamellae between adjacent gas bubbles.

Even when there is a net flow of foam in a porous medium, experiments show that foam flows in a small fraction of the pores at any given time. The rest of the pores contain trapped foam, which effectively blocks the flow. Blockage leads to a permeability that is orders of magnitude lower than for flow of a single phase. ${ }^{6}$ In fact, studies have shown that the trapped gas saturation of foams in porous media can be as high as $80 \% .^{7,8}$ Understanding the diffusion processes within trapped foams in porous media can aid in understanding the overall behavior of flowing foams in porous media.

Several methods have been developed to describe the diffusive decay of static foam in the bulk. Experimental techniques exist for measuring the rate of decay of bulk foams 9,10 and a comprehensive scaling model describing the coarsening of bulk foam was 
recently proposed by Patzek. ${ }^{11}$ However, coarsening of bulk foam is quite different from the coarsening of foam confined in a porous medium. In this work, we present the first comprehensive simulation that predicts the diffusive coarsening of static foam in a porous medium.

A model is needed to help understand how diffusion affects flowing foam systems, and to predict how stationary foam evolves with time. Below the limiting capillary pressure and in the absence of external effects, such as temperature fluctuations, evaporation, and seismic activity, foam texture coarsens as a result of diffusion of gas from bubble to bubble. The current study predicts the effect of gas diffusion and the time scales over which this effect is felt. A two-dimensional network is adopted to simulate the motion of the foam films and the variation of pressures in the foam as the diffusion process occurs. A two-dimensional network with enough connectivity does a good job of capturing all the behavior of a real system, while not being computationally limiting.

The mathematics in the simulation offer a formidable challenge. Prediction of the coarsening of foam in porous media requires the solution of a series of nonlinear, coupled differential-algebraic equations. These equations exhibit multiple singularities, at which the system passes through a series of limit points, and demonstrate multivalued solutions. In special cases, we discover oscillatory behavior of foam films as they collectively rearrange due to diffusional coarsening. To resolve the regions of mathematical difficulty, Keller's method of arc-length continuation is used, followed by a new technique that allows resolution of a solution on a separate branch.

\section{PHYSICAL PICTURE}

Foam is a dispersion of gas bubbles surrounded by thin liquid films, or lamellae, that are stabilized by surface active agents. The curvature of a lamella indicates the direction of the pressure difference between adjacent bubbles. The gas on the concave side 
of a lamella is at higher pressure than the gas on the convex side. As shown in Figure 1, in a bulk polyhedral foam, small bubbles are usually at higher pressure than the larger bubbles surrounding them. The pressure difference between adjacent gas bubbles is a function of the interfacial tension $\sigma$ of the liquid-gas interface and of the curvature of the film $(2 \mathrm{H})$, as expressed by the Young-Laplace equation,

$$
\Delta \mathrm{P}=2 \sigma(2 \mathrm{H})=4 \sigma / \mathrm{r}
$$

where $\mathbf{r}$ is the equivalent radius of curvature of the lamella. In the absence of external forces, such as gravity, a lamella is a surface of constant curvature, and the equation $2 \mathrm{H}=$ $2 / r=\left(1 / r_{1}+1 / r_{2}\right)$ holds everywhere on the surface, where $r_{1}$ and $r_{2}$ are the principal radii of curvature. The additional coefficient 2 appears in Eq. [1] because a lamella is composed of two liquid-gas interfaces. The pressure difference acts as a driving force for gas to diffuse from one bubble to the next. Therefore, large bubbles increase their volumes at the expense of smaller bubbles. Eventually, the system reaches equilibrium when the pressure is uniform everywhere. This occurs either when all bubbles are of the same size and topology, or more commonly when one large bubble remains.

Foam injected into a porous medium behaves quite differently than in bulk, due to geometric constraints at pore walls. In a water-wet porous medium, the projections of the lamellae intersect the walls at a $90^{\circ}$ angle. ${ }^{12,13}$ Therefore, the curvature of a given lamella is completely determined by its location within the pore. It is no longer necessarily true that small bubbles are at higher pressure than large bubbles. This characteristic of foam in a porous medium is illustrated by the schematic in Figure 2. The solid is water-wet, so the liquid phase forms liquid films along the grains in addition to the thin foam films.

Just as in a bulk foam, gas diffuses through the lamellae from high pressure bubbles to low pressure ones. As a result, the lamellae rearrange as the system strives to achieve a state of minimum free energy, at which point the pressures become uniform. The 
lamellae, which are assumed to be infinitely stable, move by sliding along the wetting films which coat the surface of the porous medium. This process proceeds slowly enough to allow the liquid phase to rearrange so the lamellae remain at nearly constant thickness during the process. At the equilibrium position, the lamellae all rest in the pore throats, where they have no curvature and thus sustain no pressure drop. This configuration is thermodynamically stable, and gas diffusion stops. In contrast to bulk foam, which always collapses ultimately, the equilibrium configuration of foam in a porous medium remains indefinitely in the absence of external disturbances.

The rate of transfer of gas across a lamella is described by the diffusion equation, derived from Fick's law:

$$
\frac{\mathrm{dn}}{\mathrm{dt}}=-\mathrm{k} A \Delta \mathrm{P} ; \quad \mathrm{k}=\frac{\mathrm{SD}}{\mathrm{h}}
$$

where $\frac{\mathrm{dn}}{\mathrm{dt}}$ is the number of moles of gas per unit time passing through the lamella, $\mathrm{A}$ is the surface area of the lamella across which the transfer occurs, and $\mathrm{k}$ is the mass transfer coefficient, given by the product of the solubility of the gas species in the liquid phase, $S$, and the diffusivity of the gas in the liquid phase, $D$, divided by the thickness of the film, $h$. Interfacial resistance to mass transfer can usually be neglected, as the transfer through the film is much slower than the initial dissolution of gas in the lamella 14 .

Gas diffusion in a foam in a porous medium must satisfy both the Young-Laplace equation [1] and the diffusion equation [2] for each lamella in the system. The ideal gas law relates the number of moles, $n$, to the pressures in the bubbles. The specific pore geometry chosen determines the functional dependence of radii of curvature and bubble volumes on the positions of lamellae in the porous medium. 


\section{TWO-DIMENSIONAL NETWORK MODEL}

In the simulations, the porous medium is approximated by a network consisting of a two-dimensional array of pores. Figure 3 shows a $4 X 4$ network, which is made up of 16 pore bodies, numbered 1 through 16 . Four pores come together at each pore body; each set of four pores and one body is referred to as a cluster. The clusters join together to create the network. For convenience, we divide each pore into two half-pores, so there are 64 half-pores in a $4 X 4$ network.

Each half-pore in the network is referenced by a two index system, as highlighted in pore body 1 in Figure 3. The first index $(i=1, N C)$ refers to the cluster number; the second index $(j=1,4)$ refers to the half-pore number; and $\mathrm{NC}$ is the total number of clusters in the system. Cluster 1 is in the lower right corner of Figure 3, and cluster NC is in the top left comer. Figure 3 also illustrates how the pores surrounding each pore body are numbered. We define a loop in the system as the path one follows to connect four clusters and return to the starting point. For example, in the $4 \mathrm{X} 4$ system of Figure 3 , a loop starts in pore body 2 , and passes through bodies 6,7 , and 3, traversing four pores on the way. The multiple connectivity of the pores in the network leads to interesting dynamic behavior. The system is closed, with a constant mass of gas in the system at all times. Pores along the edges of the network are treated as if there were a rigid wall at the pore throat.

To complete the network model described here, we must pick a pore geometry. The shape used in the simulator is a translationally invariant "hourglass." Two parallel cylinders of radius $R_{g}$ determine the pore cross section, as illustrated in Figure 4 . The pore width is equal to $\mathrm{R}_{\mathrm{g}}$, which is called the grain radius. The narrowest point at the center of the pore is the pore throat, where the cylinders are separated by a distance $2 r_{t}$. The space connecting pores is called the pore body. The geometry of the pore body is not important. Each pore body's volume is defined as part of the initial conditions. 
All pores are considered to be water-wet. Thus, when a lamella is located at the pore throat it has an infinite radius of curvature (zero curvature) and sustains no pressure drop. The curvature of a lamella increases as it moves away from the pore throat, and passes through a maximum between the pore throat and the pore body. This maximum in curvature occurs at the "critical position." The variable $\mathrm{x}$ represents the position of the intersection of the lamella with the pore wall, normalized by the characteristic length of the pore $\left(\mathrm{R}_{\mathrm{g}}\right) ; \mathrm{x}$ is zero at the throat and unity at the intersection of the pore with the body.

Since a lamella contacts the wall of the pore at $90^{\circ}$, it is always convex away from the pore throat. Thus, the gas in the pore throat is always at a higher pressure than that in the pore body and diffuses out of the pore. Lamellae rearrange as diffusion proceeds and ultimately recede toward the pore throats during the coarsening process. When two lamellae approach one another at the pore throat, they merge. In this way, bubbles, as defined above, completely disappear, and the foam coarsens. Coarsening occurs only when there are two lamellae located in a pore, as in Figure 4.

Knowing the pore geometry, and given the position, $x$, of a lamella in a pore and the $90^{\circ}$ contact angle of the lamella with the pore wall, the foam geometry in the system can be determined (see Appendix A). Each pore is divided conceptually into two half-pores, separated at the pore throat. Each half-pore may contain a lamella, which has a radius of curvature of

$$
\frac{r}{R_{g}}=\frac{B-\left(1-x^{2}\right)^{1 / 2}}{x}
$$

where B is a shape parameter, related to the aspect ratio. For an hourglass, B is defined as

$$
B=\left(1+\frac{r_{t}}{R_{g}}\right) \text {, }
$$


where $r_{t}$ is the radius of the pore throat, and $R_{g}$ is the radius of the grain used to generate the hourglass shape. The volume of gas inside the bubble bounded by a lamella and a pore throat is

$$
\begin{aligned}
\frac{V}{R_{g}^{3}} & =2 B x-\arcsin (x)-x\left(1-x^{2}\right)^{1 / 2} \\
& +\left(B-\left(1-x^{2}\right)^{1 / 2}\right)^{2} \frac{\left(\arccos \left(1-x^{2}\right)^{1 / 2}-x\left(1-x^{2}\right)^{1 / 2}\right)}{x^{2}}
\end{aligned}
$$

Likewise, the surface area of the lamella can be calculated,

$$
\frac{A}{R_{g}^{2}}=2 \frac{r}{R_{g}} \arccos \left(1-x^{2}\right)^{1 / 2}
$$

A fundamental difference between bulk foam and foam confined in a porous medium is that the equilibrium configuration in the porous medium is a foam which still has a substantial number of bubbles that do not necessarily have equal volumes, but do have equal pressures and zero curvature lamellae. As mentioned earlier, this is a stable configuration that remains indefinitely, assuming there are no external effects.

To start the simulation, we specify a distribution of pore sizes. For example, the grain size $R_{g}$ and shape paramter $B$ may be varied using a gaussian distribution. The distribution in grain size is defined as a function of the standard deviation in $R_{g}$ to be

$$
\psi=\frac{\overline{\mathrm{R}}_{\mathrm{g}}+\mathrm{SD}}{\overline{\mathrm{R}}_{\mathrm{g}}}
$$

where $\bar{R}_{g}$ is the average grain radius and $S D$ is the standard deviation in $R_{g}$. When $\psi \neq 1$, there is a distribution of pore volumes. In addition, we set the pore body volumes in the network such that each pore body is of the same size. Finally, we randomly distribute the lamella positions between preset limits, e.g. $0.1 \leq x \leq 0.99$. However, these positions 
cannot be random across the entire system. Each half-pore may contain a lamella, resulting in up to 8 lamellae that make up a loop in the system, as in Figure 3. Each lamella of a foam in a porous medium must satisfy the Young-Laplace equation. This means that in a loop which contains 8 lamellae, only the positions of seven of these lamellae are independent. The position of the eighth lamella is completely determined once the positions of the other seven are known. To establish an initial configuration in the simulation, seven lamellae in each loop are randomly positioned, and then the necessary radius of curvature of the eighth lamella is calculated from the Young-Laplace equation. From this radius of curvature, we calculate the corresponding lamella position,

$$
x_{i, j}=\frac{B_{i, j} \rho_{i, j} R_{g, i, j} \bar{R}_{g} \pm \sqrt{R_{g, i, j}^{4}-B_{i, j}^{2} R_{g, i, j}^{4}+\rho_{i, j}^{2} R_{g, i, j}^{2} \bar{R}_{g}^{2}}}{\left(\rho_{i, j}^{2} \bar{R}_{g}^{2}+R_{g, i, j}^{2}\right)}
$$

where $\rho_{i, j}=r_{i, j} / \bar{R}_{g}$ is the dimensionless radius of curvature of lamella $(i, j)$. Eq. [8] gives two possible solutions for the lamella position. As long as the calculated position is between 0 and 1 , we can choose either solution.

At times, Eq. [8] has no admissible solutions. Since the pressure difference is inversely proportional to the radius of curvature and there is a minimum achievable radius of curvature for any pore, sometimes it is not possible to achieve a pressure drop across the eighth lamella that is necessary to complete the loop. When this situation arises, only six of the lamellae can be randomly placed, while the two remaining lamellae positions are calculated from the Young-Laplace equation. In fact, sometimes the only admissible solution is that with an empty half-pore, leaving seven lamellae to complete the loop.

Once an acceptable initial configuration of lamellae has been created, the complete distribution of gas moles and pressures can be calculated, given the total number of moles of gas in the system. At this point, the set of equations is solved explicitly in time and the motion of all the lamellae is tracked during the coarsening. Throughout the process, the 
system is closed. No gas enters or leaves the network, and the total volume remains constant. When lamellae approach a pore throat from opposite sides, the bubble in the pore disappears as the two lamellae merge into one. In this way, there is a coarsening of foam texture from the initial configuration to the final, equilibrium state.

\section{MATHEMATICAL DESCRIPTION}

As described above, the position of each lamella in the system must satisfy Eqs. [1] and [2]. Eq. [1] is the Young-Laplace equation for a lamella which is spherical. In our translationally invariant geometry, lamellae are actually approximated by sectors of cylinders. Therefore, the curvature $2 \mathrm{H}$ is $1 / \mathrm{r}$ and $\Delta \mathrm{P}$ is $2 \sigma / \mathrm{r}$ as the second principal radius of curvature is infinite. Application of the ideal gas law relates the pressure $\mathbf{P}$ to the number of moles $n$ and the volume of gas $V$ in the Young-Laplace equation and leads to:

$$
\frac{\mathrm{n}_{\mathrm{i}, \mathrm{j}} \mathcal{R} T}{\mathrm{~V}_{\mathrm{i}, \mathrm{j}}\left(\mathrm{x}_{\mathrm{i}, \mathrm{j}}\right)}-\frac{\mathrm{n}_{\mathrm{i}} \mathcal{R} T}{\mathrm{~V}_{\mathrm{i}}\left(\mathrm{x}_{\mathrm{i}, \mathrm{k}=1,4}\right)}=\frac{2 \sigma}{\mathrm{r}_{\mathrm{i}, \mathrm{j}}}
$$

where $R$ is the universal gas constant and $T$ is temperature. A number of moles or a volume with two subscripts refers to a bubble contained in half-pore $(i, j)$. When there is one subscript, the variable refers to the value in pore body (i). Substituting the expressions for lamellar surface area, Eq. [6], and pressure drop $(\Delta \mathrm{P}=2 \sigma / r)$ into the diffusion equation [2] results in

$$
\frac{\mathrm{dn}}{\mathrm{dt}}=-4 \sigma \mathrm{k} \mathrm{R}_{\mathrm{g}} \arccos \left(1-\mathrm{x}_{\mathrm{i}, \mathrm{j}}\right)^{1 / 2}
$$

where $\frac{\mathrm{dn}}{\mathrm{dt}}$ represents the rate of gas transfer across a lamella. The equations are nondimensionalized using the average grain radius $\left(\overline{\mathrm{R}}_{\mathrm{g}}\right)$ as the characteristic length, and the 
surface tension over the characteristic length $\left(\sigma / \bar{R}_{\mathrm{g}}\right)$ to find the dimensionless pressure $\Pi$ (= $\mathrm{P} \overline{\mathrm{R}}_{\mathrm{g}} / \sigma$ ). Eqs. [9] and [10] then become the two primary residual equations,

$$
\begin{aligned}
& \frac{\eta_{i, j}}{\gamma_{i, j}\left(x_{i, j}\right)}-\frac{\eta_{i}}{\alpha_{i}\left(x_{i, k=1,4}\right)}-\frac{2}{\rho_{i, j}}=0 \\
& \frac{d \eta}{d \tau}+4 \arccos \left(1-x_{i, j}\right)^{1 / 2}=0
\end{aligned}
$$

where $\eta\left(=n R T / \sigma \overline{\mathrm{R}}_{\mathrm{g}}^{2}\right)$ is the dimensionless number of moles, $\gamma$ is the dimensionless volume of a bubble in a half-pore, $\alpha$ is the dimensionless volume of a bubble in a pore body, $\rho$ is the dimensionless radius of curvature, and $\tau$ is the dimensionless time,

$$
\tau=\frac{t D}{\bar{R}_{g}^{2} \beta} .
$$

The parameter $\beta$ is the inverse of the lamella conductivity to gas transport,

$$
\beta=\frac{h}{R T S \bar{R}_{g}} .
$$

By way of illustration, we use the $4 \mathrm{X} 4$ system in Figure 3, where cluster 2 connects to cluster 6 . More specifically, half-pore [2,2] connects to half-pore [6,3]. In this example, one lamella resides in each half-pore. When solving the problem, Eqs. [12] for adjacent half-pores are added together to give an expression which defines the total transfer of moles out of a bubble into the two adjacent pore bodies:

$$
\frac{d \eta_{2,2}}{d \tau}+\frac{d \eta_{6,3}}{d \tau}+4 \arccos \left(1-x_{2,2}^{2}\right)^{1 / 2}+4 \arccos \left(1-x_{6,3}^{2}\right)^{1 / 2}=0
$$


Since we divided each pore into two half-pores for convenience, we must constrain the pressures in the two adjacent half-pores to be equal:

$$
\frac{\eta_{2,2}}{\gamma_{2,2}\left(x_{2,2}\right)}-\frac{\eta_{6,3}}{\gamma_{6,3}\left(x_{6,3}\right)}=0
$$

In addition, lamellae $[2,2]$ and $[6,3]$ must each satisfy the Young-Laplace equation:

$$
\begin{aligned}
& \frac{\eta_{2,2}}{\gamma_{2,2}\left(x_{2,2}\right)}-\frac{\eta_{2}}{\alpha_{2}\left(x_{2, k=1,4}\right)}-\frac{2}{\rho_{2,2}}=0 \\
& \frac{\eta_{6,3}}{\gamma_{6,3}\left(x_{6,3}\right)}-\frac{\eta_{6}}{\alpha_{6}\left(x_{6, k=1,4}\right)}-\frac{2}{\rho_{6,3}}=0
\end{aligned}
$$

For every pair of half-pores, Eqs. [15-18] must be satisfied. A final constraint is the equation of mass conservation, one for each pore body, which requires that the total transfer of moles into the pore body is equal to the sum of the moles transferred out of each of the surrounding pores:

$$
\frac{\mathrm{d} \eta_{2}}{\mathrm{~d} \tau}-\sum_{\mathrm{j}=1}^{4} 4 \arccos \left(1-\mathrm{x}_{2, \mathrm{j}}^{2}\right)^{1 / 2}=0
$$

For each cluster in the network, there are eight governing equations [15-18] and one conservation equation [19] for a total of nine equations per cluster. Thus, in the $4 \times 4$ network, there are 144 total equations which completely specify the system. This system of equations forms a set of differential-algebraic equations, with first-order differential equations coupled with nonlinear algebraic equations. The nonlinearity of the algebraic equations results from substituting the expressions for volume, Eq. [5], and radius of curvature, Eq. [6], into the denominators of Eqs. [16-18]. A system with one or more 
lamellae missing has fewer equations, and some of the remaining equations have a slightly different form to account for bubbles that span more than one pore.

\section{NONLINEAR DYNAMICS}

The nine unknowns per cluster are calculated by solving the residual equations [1519], which in vector notation are:

$$
\mathbf{R}[\mathbf{u}(\tau)]=0
$$

The unknowns are the lamella positions $\left(x_{i, j}\right)$, the number of moles in each half-pore $\left(\eta_{i, j}\right)$, and the number of moles in each pore body $\left(\eta_{i}\right)$. These variables make up the solution vector $\mathbf{u}=\left[\mathrm{x}_{\mathrm{i}, \mathrm{j}}, \eta_{\mathrm{i}, \mathrm{j}}, \eta_{\mathrm{i}}\right]$. Traditionally, $(\tau)$ is the independent variable in the problem, and the residual equations [20] are solved explicitly by marching forward in time with an increment $\Delta \tau$. At each time step, the nonlinearities in the residual equations are resolved using a Newton-Raphson iteration. ${ }^{15}$ Because of the extensive coupling among the numerous lamellae, the Jacobian matrix contains a significant amount of off-diagonal elements.

The high degree of nonlinearity of the problem can lead to several solution branches. When there is a bifurcation or limit point in the solution space $\left[\mathbf{u}^{\circ} \tau\right]$, the determinant of the Jacobian $(\mathbf{G}=\partial \mathbf{R} / \partial \mathbf{u})$ passes through zero. The Newton-Raphson technique then fails because the Jacobian cannot be inverted at the singularity. Singularities arise physically due to the critical position in the relationship between radius of curvature and $\mathrm{x}$, as noted previously. Figure 5 illustrates this effect of the geometry for an hourglass with shape parameter $B=1.2$. Since the pressure drop across a lamella is inversely proportional to the radius of curvature, a maximum occurs at the critical position. In some situations, the system of lamellae in the porous medium network dictates a higher pressure 
drop across a given lamella than can be physically achieved, given the pore geometry. When this occurs, a limit point may result, and the lamellae suddenly adjust or jump to an admissible position.

We use Keller's method ${ }^{16}$ of arc-length continuation to resolve the solution around limit points. By making $\tau$ an additional dependent variable, the new solution vector is $\mathbf{v}=$ $[\mathbf{u}, \tau]$, and the new independent variable is the differential arc-length ds of the solution branch:

$$
(\mathrm{ds})^{2}=\|\mathrm{du}\|^{2}+(\mathrm{d} \tau)^{2}
$$

The system of lamellae is solved by marching explicitly in arc-length with an increment $\Delta s$, and with an additional residual equation to account for the new dependent variable,

$$
M=\theta\left\|\mathbf{u}(s)-\mathbf{u}\left(s_{0}\right)\right\|^{2}+(1-\theta)\left|\tau(s)-\tau\left(s_{0}\right)\right|^{2}-\left(s-s_{0}\right)^{2}=0
$$

where $\mathbf{u}\left(\mathrm{s}_{\mathrm{o}}\right)$ is the solution at the previous step, and $\theta \in\{0,1\}$ is an adjustable parameter. The new residual vector is $\mathbf{Q}=[\mathbf{R}, \mathbf{M}]$, and the resulting Jacobian needed for a NewtonRaphson solution is

$$
\mathbf{J}(\mathbf{s})=\left(\begin{array}{cc}
\mathbf{G} & \mathbf{R}_{\tau} \\
\mathbf{M}_{\mathbf{u}} & \mathbf{M}_{\tau}
\end{array}\right)
$$

where the new Jacobian matrix $\mathbf{J}$ is nonsingular even when $\mathbf{G}$ is singular.

Given the arc-length continuation, the tangent to the solution vector at a given step can be calculated. The tangent $\dot{v}(s)=d v(s) / d s$ satisfies

$$
\mathbf{J}(\mathbf{s}) \dot{\mathbf{v}}(\mathbf{s})=-\left(\begin{array}{c}
0 \\
\mathrm{M}_{\mathbf{s}}
\end{array}\right)
$$


The solution vector is found by iterating $\mathbf{J} \Delta \mathbf{v}=-\mathbf{Q}$ until $\mathbf{Q}=0$.

At any given time, there are multiple branches in solution space that satisfy the physical laws and contain the same number of moles within the same total volume. All the branches eventually yield equilibrium lamella configurations. However, not all branches can be reached from the initial condition because the system would have to follow a nonphysical path. To jump to the new branch, it is necessary to pick a direction to move from the point of singularity. The distance of the jump must be large enough so that the solution vector does not remain on the old branch, but yet small enough so that the new branch can be resolved.

Since the direction of the jump is not known a priori, we must devise a technique to choose the proper direction. Adjacent lamellae must move in opposite directions during the jump, because the mass inside every bubble is conserved. For example, if the lamella in half-pore $(1,1)$ moves away from the pore throat, the adjacent lamella in half-pore $(2,4)$ must follow it and move toward the throat. In addition, because a jump occurs at an instant in time, there is no diffusion of gas, and the number of moles of gas in every pore body must remain constant. Therefore, some of the lamellae surrounding each pore body move toward the body, and some move away.

We define a diagonal matrix $\Phi$ which has elements that are all close to zero, but which are seeds for the adjustments that are made to each lamella position value in the solution vector $\mathbf{u}$ in order to find the new solution branch. Since the number of moles is constant during the adjustment, we must solve for the vector of lamella positions, $\mathbf{x}$. The adjustment is the dot product of the matrix $\Phi$ and a coefficient vector $\varepsilon$, whose elements set the directions in which to make the adjustments of each lamella position and the magnitude of each adjustment. To resolve the first point on the new branch $\left(\mathbf{u}_{h}\right)$, we use

$$
\mathbf{x}_{\mathrm{h}}=\mathbf{x}\left(\mathrm{s}_{\mathrm{c}}\right)+\varepsilon \Phi
$$


to express the lamella positions in the Young-Laplace equations, where $\mathbf{x}\left(\mathrm{s}_{\mathrm{c}}\right)$ is the solution vector at the singular point. The Jacobian is made up of $\mathrm{x}$-derivatives of the YoungLaplace equations multiplied by diagonal elements of the matrix $\Phi$. The unknown vector is now $\varepsilon$, which is found by Newton-Raphson iteration and substituted into Eq. [25] to establish the new lamella positions. Once the point on the bifurcated branch is found, the rest of the branch is resolved using arc-length continuation.

\section{LAMELLA MOTION}

The net motion of all lamellae in a pore network must be toward the pore throats. However, at any given moment, an individual lamella may move away from the throat, depending on the influence of the lamellae around it. Figure 6 shows the motion of the eight lamellae in a simple $2 X 2$ network, as a function of dimensionless time ( $\tau=\mathrm{D} \beta \mathrm{t} / \overline{\mathrm{R}}_{\mathrm{g}}^{2}$ ). There is a random distribution of initial lamella positions ranging from $x=0.33$ to $x=$ 0.99. Eventually, these lamellae rest at the pore throats, where $x=0$. Six of these lamellae move in towards the pore throat at all times. However, two of them, $(2,4)$ and $(3,1)$, marked with an arrow in the figure, move away from the throats initially and then turn and head toward the throats.

When the lamella positions span the critical position, interesting behavior, such as the local reverse motion in Figure 6, results. For an hourglass with a shape parameter $\mathrm{B}=$ 1.2 , the critical position occurs when the lamella resides at $x=0.55$. When the arithmetic average of the lamella positions exceeds 0.55 , the lamellae clearly span the critical position.

In addition to the changing direction of lamella motion described above, there can be oscillatory behavior near a singular point. This type of behavior is evident in a lamellaeensemble where the initial lamella distribution is nearly uniform. In other words, each lamella is nearly the same distance from its corresponding pore throat as all the others. When the singular point is reached, near $x_{i}=0.55$, the lamellae jump to new positions in 
order to satisfy the diffusion process. In a given loop, this motion is analogous to the rotation of a "wheel." All eight lamellae in the loop rotate in the same direction, either clockwise or counterclockwise. The loop behaves like a rigid body, because the rotation happens so quickly that negligible diffusion occurs. Several rotations may occur sequentially until the system settles at a new point, at which time diffusion-driven motion continues smoothly. A 2X2 system with such an oscillation is illustrated in Figure 7. Here, all eight lamellae in the loop start at $x$ near 0.7 . Figure 7a shows that in general, the lamellae migrate toward the throat. A closer look, however, shown in Figure $7 \mathrm{~b}$, reveals an oscillation just after $\tau=48.7$.

Figure 8 graphically illustrates on an exaggerated scale what occurs as the system passes through the oscillation. The actual magnitude of the oscillation is very small, as seen in Figure 7. In Figure 8, we see four snapshots in time during the oscillation of the loop. The motions resemble the rotation of a "wheel." The dotted regions represent the solid grains. The lamellae enter the oscillation sequence in frame a, move in the direction of the arrows to the configuration in frame $b$, then turn and move back in the other direction to the configuration in frame $\mathrm{c}$, and finally go through one more jump to the configuration in frame $\mathrm{d}$. This sequence is for $48.8 \leq \tau \leq 49.5$.

When the initial lamella distribution is broader, the lamellae do not oscillate as the ones shown in Figures 7 and 8 . Instead, the system approaches a singular point and direct Newton-Raphson iteration fails. If the original solution branch is followed using arclength continuation, it is possible to continue past the singular point. It turns out that the singular point is a turning point in the solution, and a decreasing time solution can be found. Clearly, this is a nonphysical solution to the problem. As the lamellae-ensemble moves in the direction of decreasing time, gas diffuses into the pores from the pore bodies, in the direction against the pressure drop. As the system follows the nonphysical branch, many of the lamellae move away from the pore throats toward a second equilibrium position, in the pore bodies, where the lamellae have zero curvature, and diffusion across 
the lamellae stops. This is an unstable equilibrium state because any perturbation of a lamella causes that lamella to move away from the body toward the pore throat. Figure 9 is a plot of the dimensionless pressure in one of the pore bodies as a function of dimensionless time, showing the decreasing time solution when arc-length continuation is used to pass through the singular point.

Since the resolved continuation of the original branch cannot occur physically, the lamellae must jump to a new configuration that allows continuation of the physical diffusion process. In general, there is no complete oscillation as in Figure 7 , rather an instantaneous jump to a new solution branch. The jump appears to be instantaneous because our model neglects viscous losses in the system. In reality, the jump is a very rapid rearrangement of lamellae, with nearly negligible gas diffusion. The lack of a complete oscillation is due to the fact that the compressibility of the gas inside the bubbles has a damping effect on the system.

Figure 10 shows the jump from the original branch to the new branch for the system in Figure 9. Instead of continuing around the turning point, the system passes through the jump described in the previous section. The bubbles move as nearly rigid bodies because the gas does not redistribute. Their pressures do change slightly, so the bubbles compress or relax during the rearrangement. In fact, in a pore with $R_{g}=200 \mu \mathrm{m}$ and a system with a surface tension of 35 dyne $/ \mathrm{cm}$, the dimensionless pressure jump shown in Figure 10 corresponds to an actual change in pressure of $21.2 \mathrm{~Pa}\left(2.1 \times 10^{-4}\right.$ atm), which is very small. In general, the loops move as rotating wheels, as illustrated in Figure 11, where the arrows show the direction of the jump from frame $a$ to $b$.

Systems larger than $2 \mathrm{X} 2$ exhibit more complicated behavior at a singular point, as not all of the loops behave like a wheel. The criterion of no mass transfer out of each bubble during the sudden rearrangement must still be met. Figure 12a shows a 3X3 network with arrows sketched in the shaded grains showing the direction of "wheel" rotation for each of the 4 loops during the jump. Notice that the loop at the top left actually 
divides into two halves which rotate in opposite directions. Figure $12 \mathrm{~b}$ shows a close up of the cluster marked with an asterisk in Figure 12a, with the lamellae sketched and arrows showing the direction of motion of these lamellae during the jump. The mass of gas in the bubble in the pore body marked with an asterisk is conserved because the motion toward the body from the bottom is balanced by the sum of the motions out of the body to the top and right. The lamella on the left in Figure $12 \mathrm{~b}$ does not move during the jump because it is on an outer boundary and thus does not interact with other lamellae.

As a lamellae-ensemble decays to its final equilibrium state, several transitions or turning points may be encountered. In fact, the $4 X 4$ network system illustrated in Figures 9 to 11 reaches a second transition point where the system undergoes a second rearrangement shown in Figure 13. After this point, the system decays until reaching the low-free energy equilibrium configuration.

It is useful to verify that the free energy of the system decreases with time during the diffusion-driven rearrangement of the lamellae. To describe a system of constant volume and temperature, we use the Helmholtz free energy. The Helmholtz free energy is

$$
F-F^{0}=-\sum_{i} p_{i} V_{i}+\sigma \sum_{i} A_{i}+R T \sum_{i} n_{i} \ln \left(\frac{P_{i}}{N R T / V_{t}}\right)
$$

where $\mathrm{N}$ is the total number of moles of gas in the system, and $\mathrm{V}_{\mathrm{t}}$ is the total system volume. The summations in the equation indicate that we must sum the contributions of every bubble in the system, both in the pore throats and the pore bodies. The reference energy $\mathrm{F}^{\circ}$ is the equilibrium Helmholtz energy. Also, we must remember that each lamella contributes twice to the surface area term, one for each liquid-gas interface. After nondimensionalizing Eq. [26] using the scaling in presented above, we verify in Figure 14 that the Helmholtz free energy indeed decreases for a single lamella in a pore as it moves towards equilibrium. 
Figure 15 shows the overall behavior of the Helmholtz free energy in a $3 \times 3$ network during the rearrangement of the lamellae-ensemble. The energy decreases throughout the entire process. This system passes through one transition point, and $\mathrm{F}$ decreases during the jump to the new configuration as well.

\section{RESULTS AND DISCUSSION}

The equilibrium pressure in a pore body must be higher than its initial pressure. This is true because the lamellae that surround the pore bodies encapsulate gas at higher pressure in the pore throats than in the pore bodies. As the gas escapes from the pore throats across the lamellae during diffusion, the pressures in the pore bodies increase. This is analogous to the increase in pressure of a headspace above a bulk foam, which has been verified experimentally ${ }^{17,18}$ and is predicted by available models. ${ }^{9,11}$ Individual pore bodies may experience decreases in pressure initially, depending on how they interact with the other pore bodies in the network, but they always end up at a higher pressure than initially. In fact, all pore bodies end up at the same pressure. However, the average pore body pressure must increase at all times. These results are verified in Figure 16 for a $5 \times 5$ system, where the diffusion process reaches equilibrium. Recall that the pressure is nondimensionalized by $\left(\sigma / \overline{\mathrm{R}}_{\mathrm{g}}\right)$, which is the characteristic pressure for a Young-Laplace problem and represents the degree of encapsulation of pressure in the system.

For diffusion collapse of a single spherical foam bubble, Chambers ${ }^{19}$ derived from the diffusion equation [2] the characteristic diffusion time. The length of time it takes a spherical bubble, in contact with an infinite medium, to disappear is approximated as

$$
t_{d i f}=h P_{0} R_{0}^{2} / 8 \text { D S R T } \sigma
$$


where $R_{0}$ is the initial bubble radius and $P_{0}$ is the initial bubble pressure. The initial bubble pressure is approximately equal to the pressure outside the bubble, since the YoungLaplace correction ( $4 \sigma / \mathrm{R}_{0}$ ) is much less than one atmosphere. For a single bubble of radius $150 \mu \mathrm{m}$ at atmospheric pressure, the diffusion time is about $1 \mathrm{~min}$. The constants used in this calculation are the ones used throughout this paper: $D=1 \times 10^{-9} \mathrm{~m}^{2} / \mathrm{s} ; \mathrm{S}=$ $5 \times 10^{-6} \mathrm{~mol} /\left(\mathrm{m}^{3} \mathrm{~Pa}\right)$; and $\sigma=3.5 \times 10^{-2} \mathrm{~N} / \mathrm{m}$.

We seek to establish the characteristic time for the diffusion process in a porous medium. This is the time for a single lamella to move from its initial position to its final resting place in the pore throat, with zero curvature. For a single bubble with an initial radius of curvature of $150 \mu \mathrm{m}$ in a pore with a throat radius of $43 \mu \mathrm{m}\left(\mathrm{R}_{\mathrm{g}}=216 \mu \mathrm{m}\right)$ at atmospheric pressure, the simulation gives an elapsed time to reach equilibrium of about 11 min. Accordingly, a bubble in a porous medium takes an order of magnitude longer to decay than a bubble in bulk with the same initial radius and pressure.

The characteristic time for diffusion of a bubble in a porous medium is given by

$$
\mathrm{t}_{\mathrm{c}}=\frac{\overline{\mathrm{R}}_{\mathrm{g}}^{2}}{\mathrm{D}} \beta \bar{\Pi}_{\mathrm{c}}
$$

where $\bar{\Pi}_{c}$ describes the amount of gas initially encapsulated in the pore throats. The measure of encapsulation is the average initial number of moles of gas $\bar{n}$ in the bubbles spanning the pore throats, converted to a pressure and nondimensionalized by the surface tension over characteristic length,

$$
\bar{\Pi}_{\mathrm{c}}=\frac{\overline{\mathrm{n} R T}}{\overline{\mathrm{R}}_{\mathrm{g}}^{3}} \frac{\overline{\mathrm{R}}_{\mathrm{g}}}{\sigma}
$$

Eq. [14] defines the parameter $\beta$, which describes the resistance to transfer of gas across a lamella from the pore throat to the pore body. The lower the value of $\beta$, the faster 
is the gas transport across the lamellae. For a thicker lamella or lower solubility, the rate of diffusion will decrease.

The characteristic time for a bubble with a radius of curvature of $150 \mu \mathrm{m}$ in a pore with a throat radius of $43 \mu \mathrm{m}$ is calculated from Eq. [28] to be almost $10 \mathrm{~min}$. This illustrates the order of magnitude difference in time scale between diffusion of bulk foam and foam in a pore and is very close to the $11 \mathrm{~min}$. decay time found in the simulation.

The characteristic time, and thus the time for the system to reach equilibrium, scales

as $\bar{\Pi}_{\mathfrak{c}}$, which is in turn linearly proportional to the system pressure. The system pressure is changed in the simulation by changing the total number of moles of gas in the network. The dimensionless variable which represents the total number of moles of gas is

$$
v=\frac{N R T}{\bar{R}_{g}^{2} \sigma} .
$$

Figure 17 shows how the decay time changes with varying system pressures for $3 \times 3$ networks. Each of these systems has the same total network volume and the same initial lamella configuration, the only difference being the total number of moles of gas distributed in the network $(v)$, and thus the final system pressure. We define the decay time, $t_{e q}$, as the time at which the diffusion process is ninety percent of the way to equilibrium, based on the average lamella position being at $10 \%$ of its initial value. Figure 17 is important for the application of foam at reservoir conditions, which are typically at very high pressures. Thus, the diffusion time for foam is long, as much as several hours for systems at $10 \mathrm{~atm}$ and with an average pore size of $100 \mu \mathrm{m}$.

The decay time for diffusion of foam in a porous medium depends strongly on the size of the pores. Smaller size pores result in more tightly curved lamellae; thus, the driving force for diffusion is greater and the foam completes the diffusion process faster. 
In fact, Eq. [28] shows that the characteristic time for diffusion is proportional to the characteristic pore size for constant pressure.

The most important consequence of scaling time by $\bar{\Pi}_{c}$ is that the time for the system to reach equilibrium is independent of network size. For example, a $2 \times 2$ system, which has 4 clusters, takes the same reduced time to complete the diffusion process as a $7 \mathrm{X} 7$ system, with 49 clusters, as long as the lamella distribution is the same. In addition, the dimensionless pressure increase is the same for different size networks with identical lamella distributions. Therefore, we can understand the behavior of a megascopic porous medium by scrutinizing the smaller systems. This results in significant computational savings.

End effects have a slight impact on the quality of the results for smaller systems. End effects arise because the pores in between two pore bodies may have two lamellae in them and definitely have interactions with multiple pore bodies. The pores on the sides of the network only interact with one pore body. Smaller networks clearly have a higher fraction of the system made up of pores which only connect to one pore body. An even bigger disadvantage of a small network is the inability to set a truly random initial lamella distribution with the smaller number of pores. For example, when there are only 36 or 64 lamellae, like in a $3 X 3$ or $4 X 4$ network, the distribution is less random than it is in a system with 1000 lamellae.

The scaling arguments for pressure and time can be summarized in "universal" curves of pore body pressure increase as a function of time. Pore body pressure increase represents how much the pore body pressure deviates from the initial value $\left(\Pi_{0}\right)$. For identical lamella position distributions, a universal curve for all absolute pore sizes, all system pressures, and all values of the parameter $\beta$ can be achieved by normalizing pressure by $\sigma \bar{R}_{\mathrm{g}}$ and normalizing time by $t_{c}$. The universal curve for one particular lamella distribution, a uniform pore size distribution, and shape parameter $B=1.2$, is given for a 
3X3 network in Figure 18. The plot shows the universality of the curve, even for systems with varying pressures $(v)$, pore sizes $\left(R_{g}\right)$, and solubility parameters $(\beta)$.

Increasing the pore body volumes decreases the final value of normalized pressure in Figure 18 because the ratio of encapsulated pressure to pore body pressure decreases. However, the effect is minimal, and variations in the distribution of pore body volumes are not important in the overall behavior of the system.

If we plot the average lamella position as a function of the reduced time, the resulting curve is also universal. Figure 19 shows this result for the same data presented in Figure 18.

The universal curve is the same for different pore size distributions. The pore size distribution is changed by adjusting the standard deviation of the pore characteristic lengths $\left(R_{g}\right)$ and by changing the range within which $B$ falls. Figure 20 shows the normalized plot of pressure versus time for various pore size standard deviations and different values of $B$. The normalized lamella plot is given in Figure 21. The agreement is not as good here as it was before. This is because changing these distributions results in slightly different lamella distributions, and therefore the initial diffusion driving forces and amounts of encapsulated pressure are different. Also, the final pressures differ slightly in Figure 20 because the total system volumes differ as the B values differ. Nevertheless, we have discovered a way of universally determining the diffusion behavior of foam in porous media by using scaling constants, as long as the lamella distribution for the system is fixed. This behavior is similar to that in the numerical solutions of Herdtle and Aref $^{20}$ where different initial bubble size distributions in bulk foam resulted in different rates of convergence to the universal self-similar solution.

The final value of pressure is different in Figure 20 than in Figure 18. This is because the initial lamella configuration is different. The final pressure value depends on the ratio of volume inside the bubbles spanning the pore throat to the volume in the pore bodies. The higher this ratio, the higher the final pressure. 
The requirement that the lamella distribution be fixed is, of course, hard to meet when trying to simulate a real system since we never know the actual lamella distribution. In order to predict accurately the behavior of real systems, we must use large networks so that differences in the random generation of lamella positions will be smaller. Then we must average the results obtained from several different runs, each with different, random configurations of lamellae.

The network simulation can be solved with a coordination number of 4 or 6 , representing the number of pores that connect at a single pore body. Both give the same overall and local behavior. Furthermore, it is easy to substitute the equations which define a three-dimensional hourglass in place of the ones for a translationally invariant hourglass. Again, this substitution does not change the results of the simulation. Both of these tests make us comfortable with the use of the two-dimensional model with a coordination number of 4 to simulate foam in a full porous medium. Clearly, the two-dimensional case is less computationally intensive, and its use is, therefore, desirable.

\section{SUMMARY}

A two-dimensional network simulation model was created to predict the behavior of foam in a porous medium. The model generates a nearly random initial configuration of lamellae in the pores, which are defined as water-wet translationally invariant hourglass shapes. Four pores are connected together in a cluster at a single pore body. Once the initial configuration is generated, the simulation tracks the motion of the individual lamellae as they rearrange in the porous medium. During this process, all the bubble pressures are calculated and the change in the average system pressure is investigated. The pressure trends predicted can be compared with experimental measurements of gas pressures in porous media. 
The time scale for diffusion is an order of magnitude greater for a bubble at atmospheric pressure in a porous medium than for the same size bubble in bulk. The time to reach equilibrium is a quadratic function of the characteristic pore size and a linear function of pressure. At high pressure, the foam takes a much longer time to decay. The time scale is independent of network size.

The lamellae in a porous medium recede toward the pore throats during the diffusion process. The pressures of the bubbles in the pore bodies increase to equilibrium. The magnitude of the pressure increase is inversely proportional to the characteristic pore size. When scaled properly, the data for pressure as a function of time and average lamella position as a function of time fall on a universal curve. There is one universal curve for each initial lamella configuration.

The curvature of the pore walls leads to interesting behavior caused by the presence of a critical position, where a lamella has a zero derivative in its radius of curvature with respect to position. This position corresponds to a minimum value of the radius of curvature and therefore a maximum value of the pressure drop across the lamella. The critical position leads to singular behavior of the system. When this occurs, the lamellae must either jump from their current configuration to another solution branch or they oscillate. At a singular point, all the lamellae in a loop must move in a cooperative way, since the jump happens so quickly that negligible gas diffusion occurs.

The techniques presented in this paper can be used to predict how foam bubbles in a porous medium of arbitrary size change as a result of diffusion. While this diffusive behavior does not have a major effect on the overall lifetime of the foam, it does result in a rearrangement of foam in a porous medium. The texture of foam changes in that the number of lamellae remaining trapped in the porous medium is reduced. The equilibrium state is one where all bubbles are at equal pressure. Understanding the diffusion evolution is particularly important if one is trying to characterize the region of trapped foam in a system where foam is flowing through a porous medium. 


\section{Symbols}

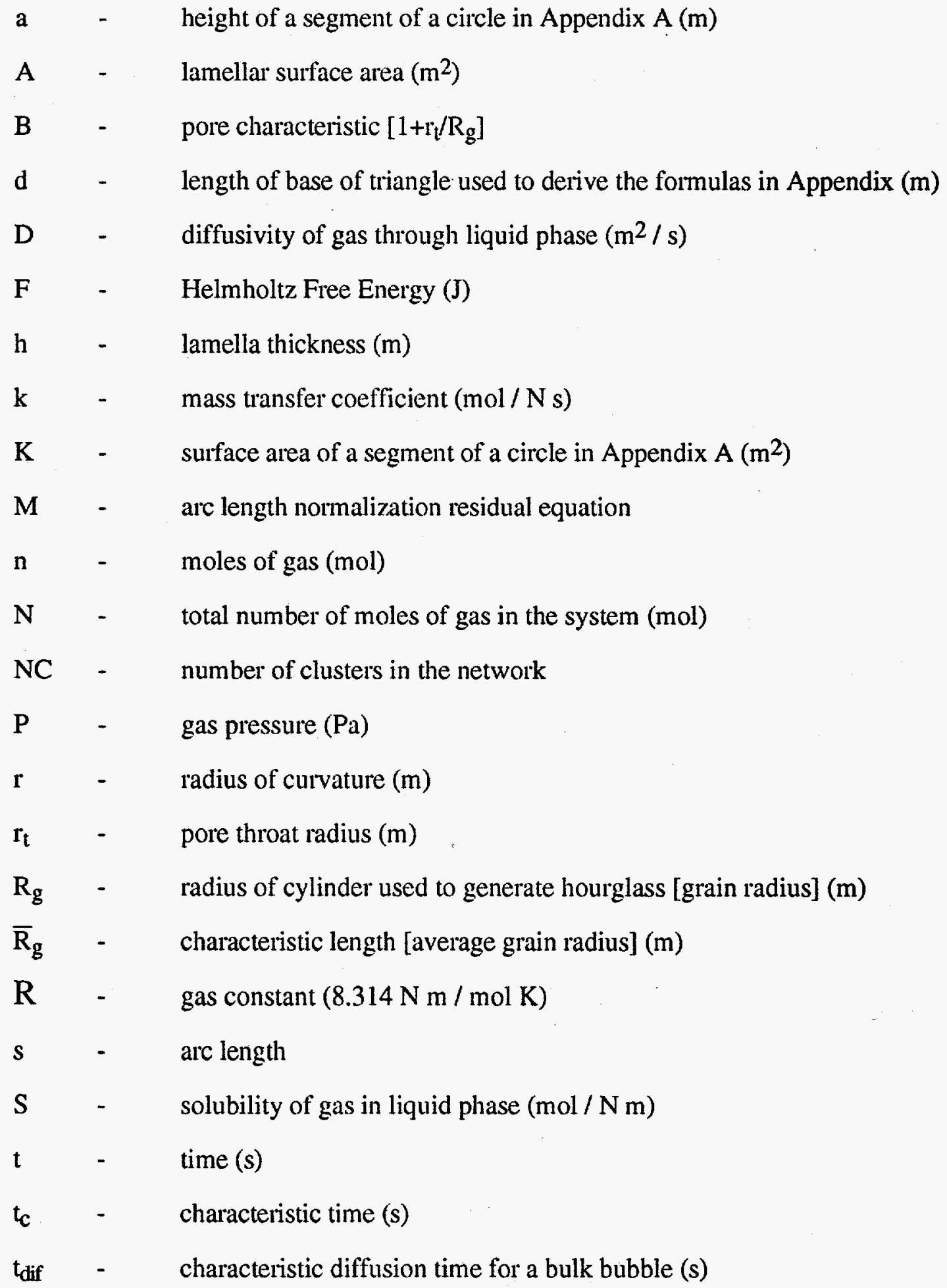




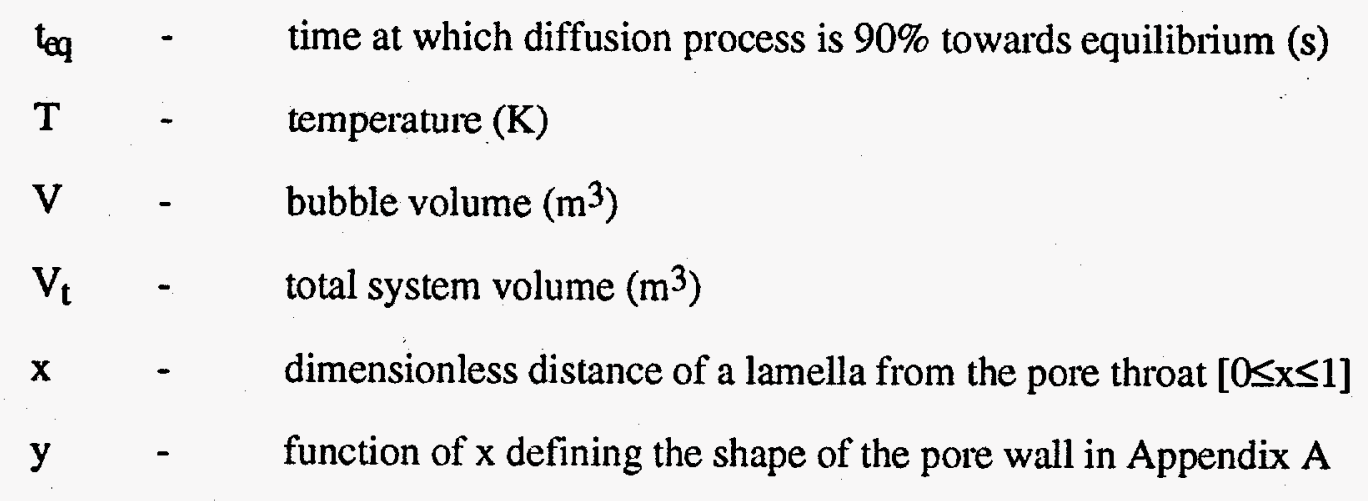

\section{Greek letters}

$\alpha$ - dimensionless pore body volume

$\beta \quad$ - solubility parameter $\left[\mathrm{h} / \mathrm{RTS} \overline{\mathrm{R}}_{\mathrm{g}}\right]$

$\gamma \quad$ - dimensionless volume $\left[\mathrm{V} / \overline{\mathrm{R}}_{\mathrm{g}}{ }^{3}\right]$

$\checkmark \quad-\quad$ dimensionless total number of moles of gas [NRT/ $\left./ \overline{\mathrm{R}}_{\mathrm{g}}{ }^{2}\right]$

$\eta \quad$ - dimensionless number of moles of gas $\left[\mathrm{nRT} / \sigma \overline{\mathrm{R}}_{\mathrm{g}}{ }^{2}\right]$

$\Pi \quad$ - dimensionless pressure $\left[\mathrm{P}_{\mathrm{g}} / \sigma\right]$

$\bar{\Pi}_{c} \quad$ - dimensionless measure of encapsulation $\left[n R T / \sigma \bar{R}_{g}{ }^{2}\right]$

$\rho \quad$ - dimensionless radius of curvature $\left[r / \bar{R}_{\mathrm{g}}\right]$

$\sigma \quad-\quad$ liquid/gas interfacial tension $(\mathrm{N} / \mathrm{m})$

$\tau$. dimensionless time $\left[\mathrm{tD} / \overline{\mathrm{R}}_{\mathrm{g}}{ }^{2} \beta\right]$

$\theta \quad$ - arc length normalization parameter

$\psi \quad$ - grain size distribution factor - sum of $\overline{\mathrm{R}}_{\mathrm{g}}$ and its standard deviation over $\overline{\mathrm{R}}_{\mathrm{g}}$

\section{Subscripts}

$\begin{array}{lll}\mathbf{i} & - & \text { cluster index }\{\mathrm{i} \in(1, \mathrm{NC})\} \\ \mathbf{j} & - & \text { pore index }\{\mathrm{j} \in(1,4)\} \\ \mathbf{0} & - & \text { initial value }\end{array}$

Vectors and Matrices 


$\begin{array}{lll}\mathbf{G} & - & \text { Jacobian matrix - derivative of } \mathbf{R} \text { with respect to elements of } \mathbf{u} \\ \mathbf{J} & - & \text { augmented Jacobian matrix (arc-length continuation) } \\ \mathbf{Q} & - & \text { augmented residual vector (arc-length continuation) }[\mathbf{R}, \mathbf{M}] \\ \mathbf{R} & - & \text { residual vector } \\ \mathbf{u} & - & \text { solution vector }\left[\mathrm{x}_{\mathbf{i}, \mathrm{j}}, \eta_{\mathrm{i}, \mathrm{j}}, \eta_{\mathrm{o}, \mathrm{i}}\right] \\ \mathbf{v} & - & \text { augmented solution vector }[\mathbf{u}, \tau] \\ \boldsymbol{\varepsilon} & - & \text { coefficient adjustment vector } \\ \mathbf{\Phi} & - & \text { diagonal matrix, seed for jumping to new branch }\end{array}$

\section{APPENDIX A - Derivation of the Mensuration Formulas}

We start from the equation for a circle of radius $R_{g}$. The center of the circle is above the origin by a distance $R_{g}+r_{t}$ where $r_{t}$ is the throat radius. The dimensionless equation for the circle is

$$
y(x)=B-\left(1-x^{2}\right)^{1 / 2}
$$

where B is the shape parameter defined in Eq. [4]. The expression for radius of curvature of a lamella at position $\mathrm{x}$ is derived by drawing a right triangle whose base is the center line of the pore and whose hypotenuse is the tangent line to the pore wall at the point where the lamella intersects the pore wall. The third side of the triangle is the line segment of length $y(x)$ connecting the point where the lamella intersects the pore wall with the center line. The length of the hypotenuse of the triangle is the radius of curvature of the lamella. The expression for radius of curvature is given in Eq. [3].

The surface area of a lamella per unit width of translationally invariant hourglass, Eq. [6], is calculated using the formula for the length of a segment of a circle, 


$$
A=2 r \arccos (d / r),
$$

where $d$ is the length of the base of the triangle described above.

There are two parts that make up the expression for volume per unit length of a bubble in a pore. The first is the surface area of the pore itself from 0 to $\mathrm{x}$. The second is the surface area of the circular cap which is defined by the lamella. To get the first part, it is necessary to integrate Eq. [A1] from 0 to $x$. The second part involves the mensuration formula for the surface area of a segment of a circle,

$$
\mathrm{K}=\mathrm{r}^{2} \arccos [(\mathrm{r}-\mathrm{a}) / \mathrm{r}]-(\mathrm{r}-\mathrm{a})\left(2 \mathrm{ra}-\mathrm{a}^{2}\right)^{1 / 2}
$$

where $a$ is the height of the segment. For our geometry, $a$ is the distance along the center line from the position $x$ to the lamella. These two parts are summed together to give the expression for volume in Eq. [5]. 


\section{References}

(1) Fried, A. N. "The Foam-Drive Process for Increasing the Recovery of Oil," U.S. Bureau of Mines, 1961.

(2) Patzek, T. W. SPE Res. Eng. 1996, 11.

(3) Persoff, P. J.; Radke, C. J.; Pruess, K.; Benson, S. M.; Witherspoon, P. A. Paper presented at SPE California Regional Meeting, Bakersfield, CA 1989.

(4) Hanssen, J. E. Journal of Petroleum Science and Engineering 1993, 10, 117156.

(5) Khatib, Z. I.; Hirasaki, G. J.; Falls, A. H. SPE Resv. Eng. 1988, 3, 919-926.

(6) Prieditis, J.; Flumerfelt, R. W. In Surfactant-Based Mobility Control; D. H. Smith, Ed.; American Chemical Society: Washington DC, 1988; Vol. 373; pp 295326.

(7) Gillis, J. V. Ph. D. Thesis, University of California at Berkeley, 1990.

(8) Friedmann, F.; Chen, W. H.; Gauglitz, P. A. SPE Res. Eng. 1991, 6, 37-45.

(9) Nishioka, G.; Ross, S.; Whitworth, M. Journal of Colloid and Interface Science $1983,95,435-442$.

(10) Monsalve, A.; Schechter, R. Joumal of Colloid and Interface Science 1983, 97, 327-334.

(11) Patzek, T. W. AlChE Joumal 1993, 39, 1697-1707.

(12) Bikerman, J. J. Foams, ed.; Springer-Verlag: Berlin, 1973.

(13) Chambers, K. T.; Radke, C. J. In Interfacial Phenomena in Petroleum Recovery; N. R. Morrow, Ed.; Marcel Dekker, Inc.: New York, 1990; pp 191253.

(14) de Vries, A. J. Recueil 1958, 77, 209-461.

(15) Press, W. H.; Vetterling, W. T.; Teukolsky, S. A.; Flannery, B. P. Numerical Recipes in Fortran , 2nd ed.; Cambridge University Press: New York, 1992. 
(16) Keller, H. B. In Applications of Bifurcation Theory; P. H. Rabinowitz, Ed.; Academic Press: New York, 1977; pp 359-384.

(17) Nishioka, G.; Ross, S. Journal of Colloid and Interface Science 1981, 81, 1-7.

(18) Schechter, R. S.; Monsalve, A. Journal of Colloid and Interface Science 1984, 97, 327-335.

(19) Chambers, K. T. M. S. Thesis, University of California, Berkeley, 1990.

(20) Herdtle, T.; Aref, H. Journal of Fluid Mechanics 1992, 241, 233-260. 


\section{Figure Captions}

Figure 1. Photograph of a bulk foam. Notice that the lamellae are curved toward the larger bubbles. The arrows represent the direction of gas diffusion. Large bubbles grow at the expense of small bubbles.

Figure 2. Schematic of foam in a porous medium. The cross-hatched regions represent the grains of the porous medium. The dotted regions are the liquid phase, which form thin films along the solid and lamellae spanning the pores. Shaded regions indicate the gas phase.

Figure 3. A $4 X 4$ network illustrating the indexing system. The solid matrix of the porous medium is represented by dashed regions, and the lamellae are represented by curved lines.

Figure 4. Translationally invariant hourglass pore with a lamella at normalized position $\mathbf{x}$.

Figure 5. (a) Critical position in radius of curvature and (b) pressure difference. The inflection point of the radius of curvature corresponds to a maximum allowable pressure drop across a lamella in the pore.

Figure 6. Motion of eight lamellae in a simple $2 \mathrm{X} 2$ network with $\mathrm{B}=1.2$ and $\psi=1$. All lamellae migrate toward the pore throat, but the lamellae in pores $(2,4)$ and $(3,1)$, marked by the arrow, move in the opposite direction initially.

. Figure 7. (a) Lamella positions in a $2 \times 2$ network with a narrow lamella distribution. Looking closely at the circled region of the graph, (b) shows the oscillation that occurs. 
Figure 8. Schematic illustration of oscillation in Fig. 7 on an exaggerated distance scale.

Figure 9. Dimensionless pressure in a pore body versus dimensionless time for a $4 \mathrm{X} 4$ network. The solution continues around the turning point and heads back in time. The lower branch is nonphysical.

Figure 10. Dimensionless pressure in a pore body versus time for a $4 \times 4$ network, the same system as in Figure 9. The solution jumps to a new branch at the turning point.

Figure 11. Rotation of "wheel" in a $2 \times 2$ network at a singular point. a) The lamellae hit the turning point and jump in the direction of the arrows to the configuration in b).

Figure 12. (a) Lamella motion during jump at a singular point in a $3 \times 3$ network. (b) Closer look at the behavior of the lamellae around the pore body marked with an asterisk. The mass in each region is conserved.

Figure 13. Dimensionless pressure in a pore body versus time for the $4 X 4$ network of Figures 9 and 10. The solution passes through two transition points, as marked by arrows in the figure.

Figure 14. Helmholtz free energy and lamella position versus time for a single lamella sitting in a pore $(B=1.2)$.

Figure 15. Helmholtz free energy versus time for a $3 \times 3$ network system. F decreases at all times. 
Figure 16. Pressure change in the pore bodies during diffusion in a $5 \times 5$ network. One individual pore body pressure is plotted, as is the average value of all the pore body pressures.

Figure 17. Linear dependence of completion time on system pressure.

Figure 18. Universal curve of pressure, normalized by $\sigma / \bar{R}_{g}$, plotted against time, normalized by $t_{c}$. All four systems have a uniform pore size distribution and the same initial lamella distribution. The absolute grain size varies in these systems by 5 orders of magnitude, as does the parameter $\beta$.

Figure 19. Universal curve of average lamella position, normalized by its corresponding pore size, plotted against time, normalized by $t_{\mathfrak{c}}$. Same systems as in Figure 18.

Figure 20. Universal curve of pressure, normalized by $\sigma / \bar{R}_{\mathrm{g}}$, plotted against time, normalized by $t_{c}$, for various distributions of geometric parameters.

Figure 21. Universal curve of average lamella position, normalized by its corresponding pore size, plotted against time, normalized by $t_{c}$, for various distributions of geometric parameters. Same systems as in Figure 20. 


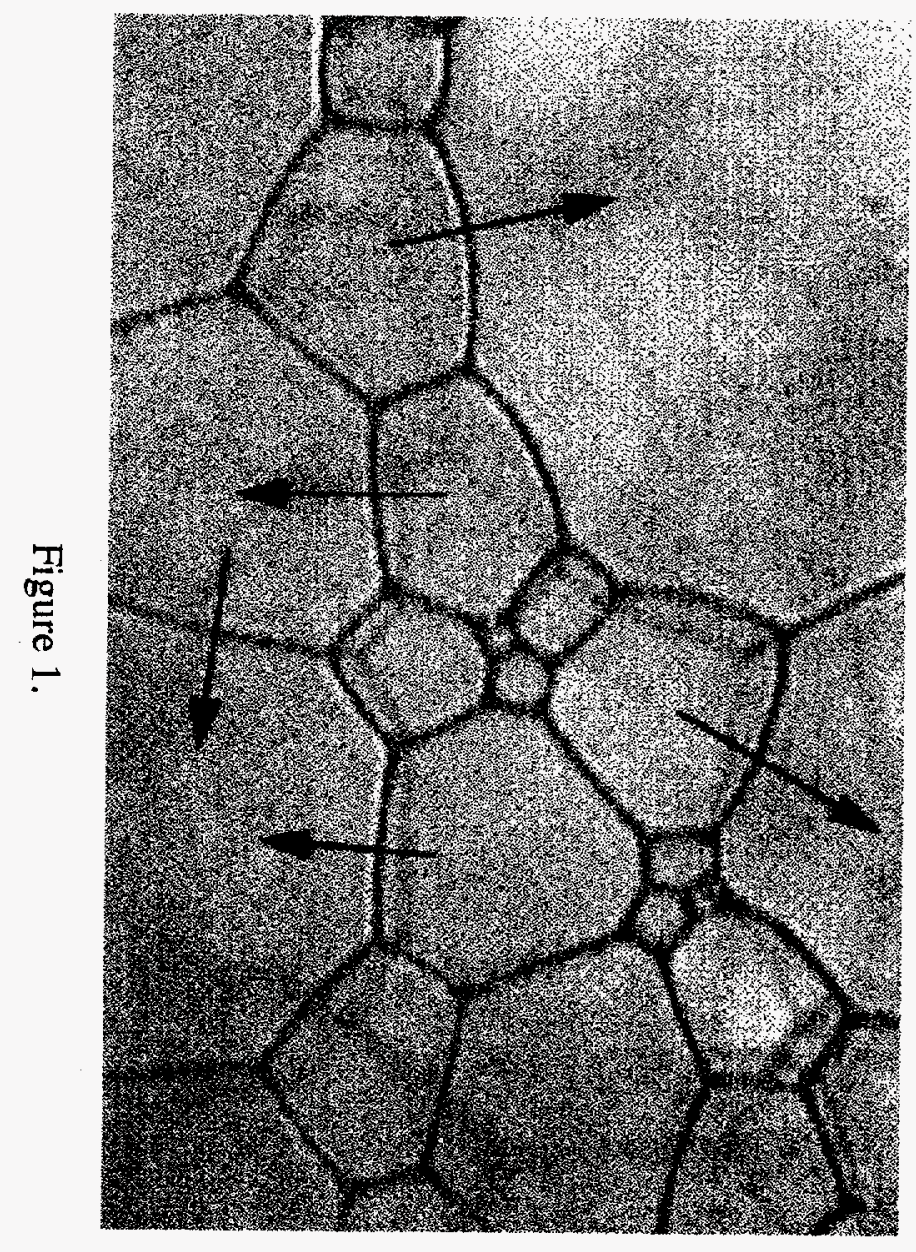




$$
\text { 뎝 }
$$




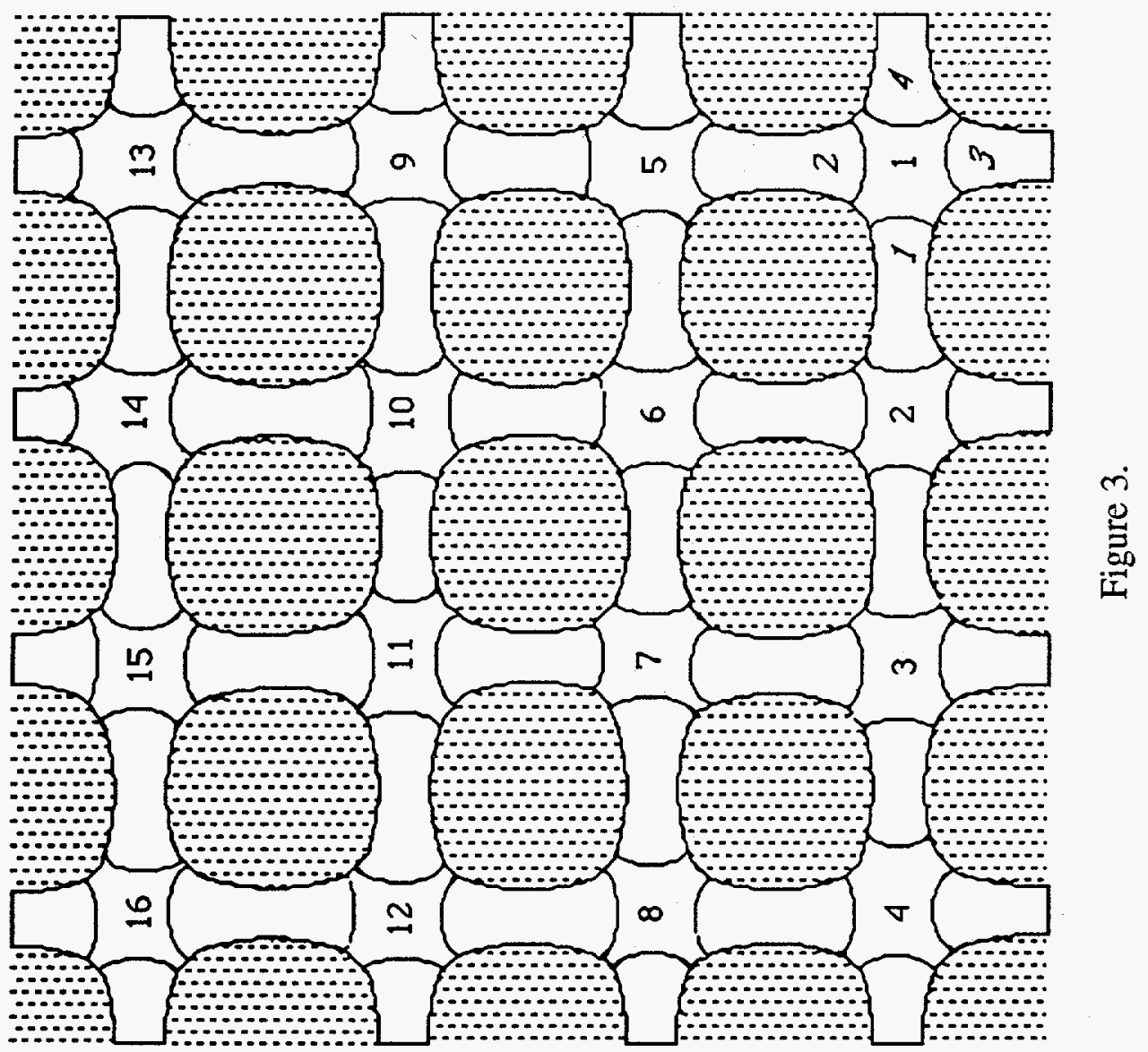




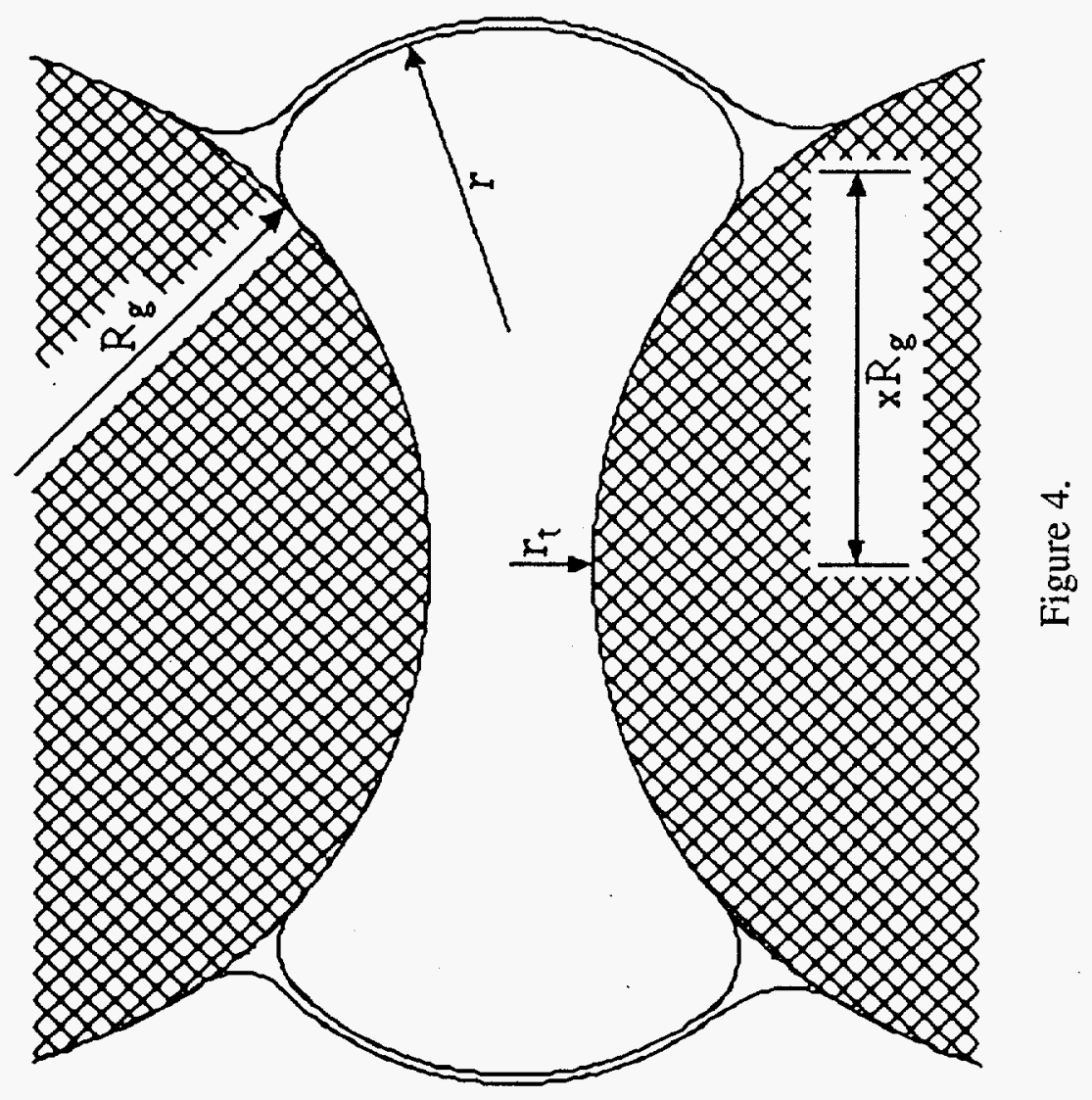



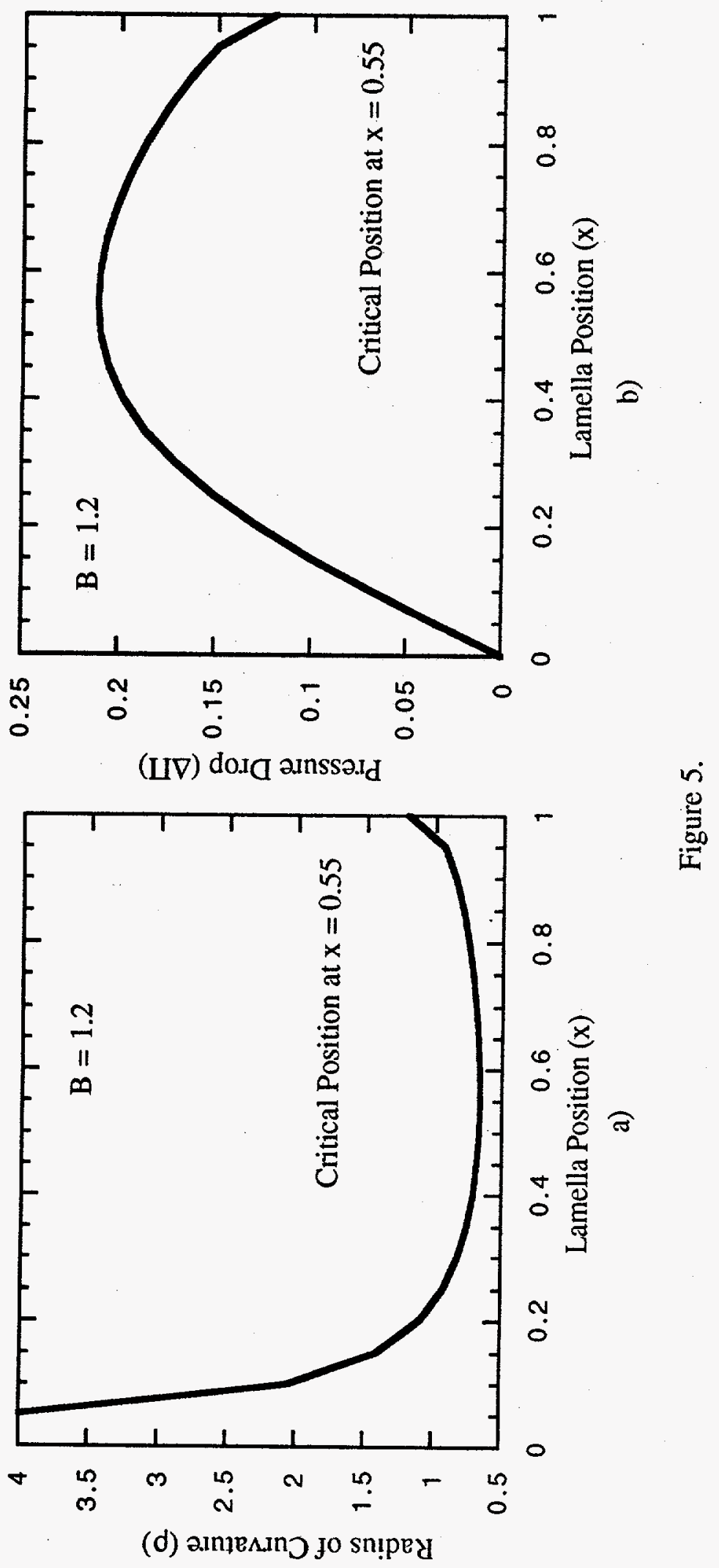


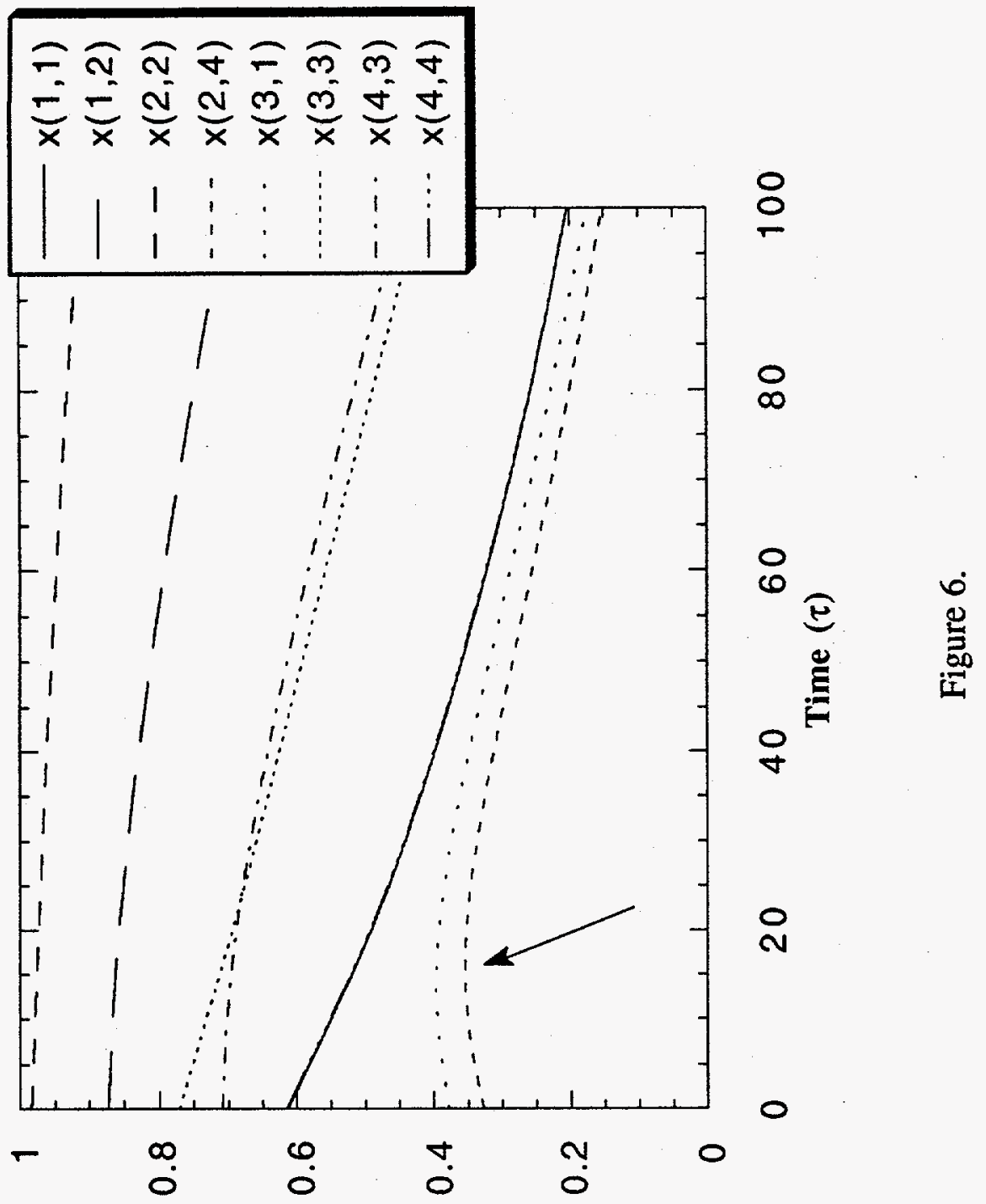

(x) uo!nsod el|วue'T 


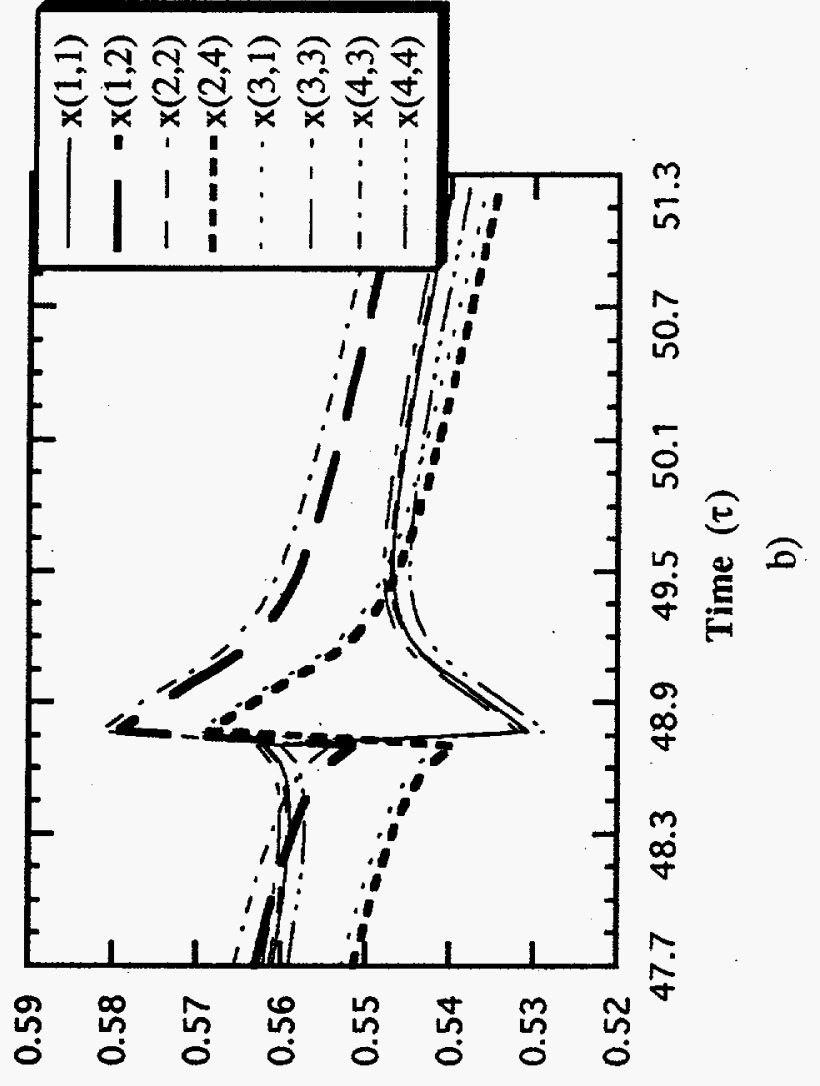

(x) uoṇ!sod rl|əureT

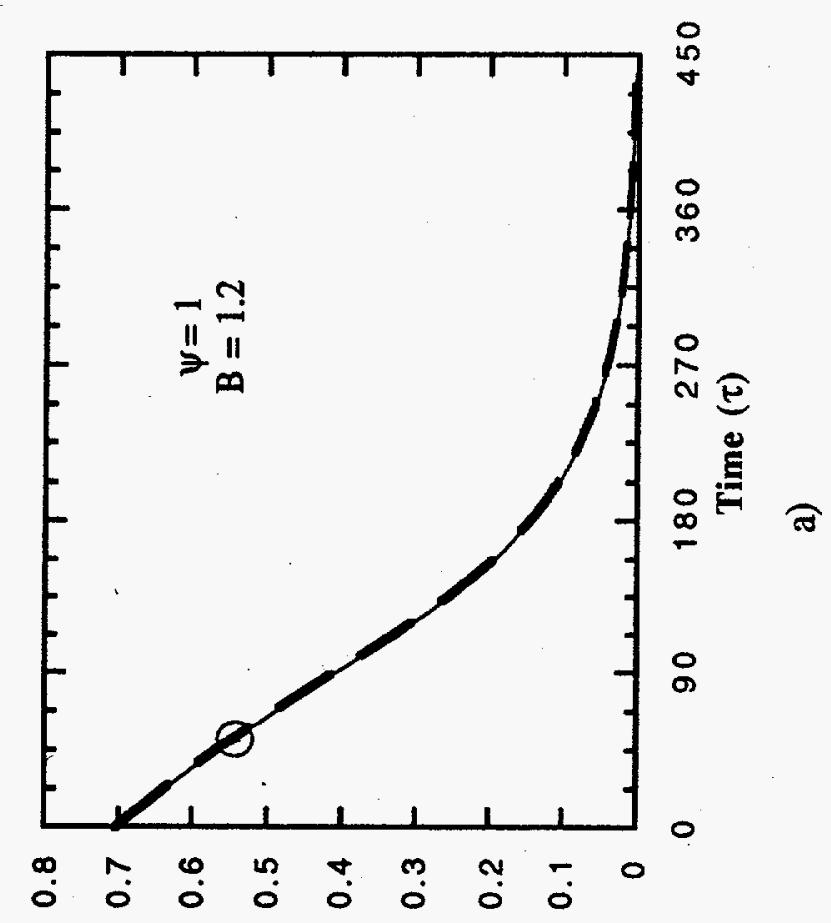

(x) uo!nsod ellaweT 


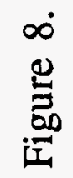
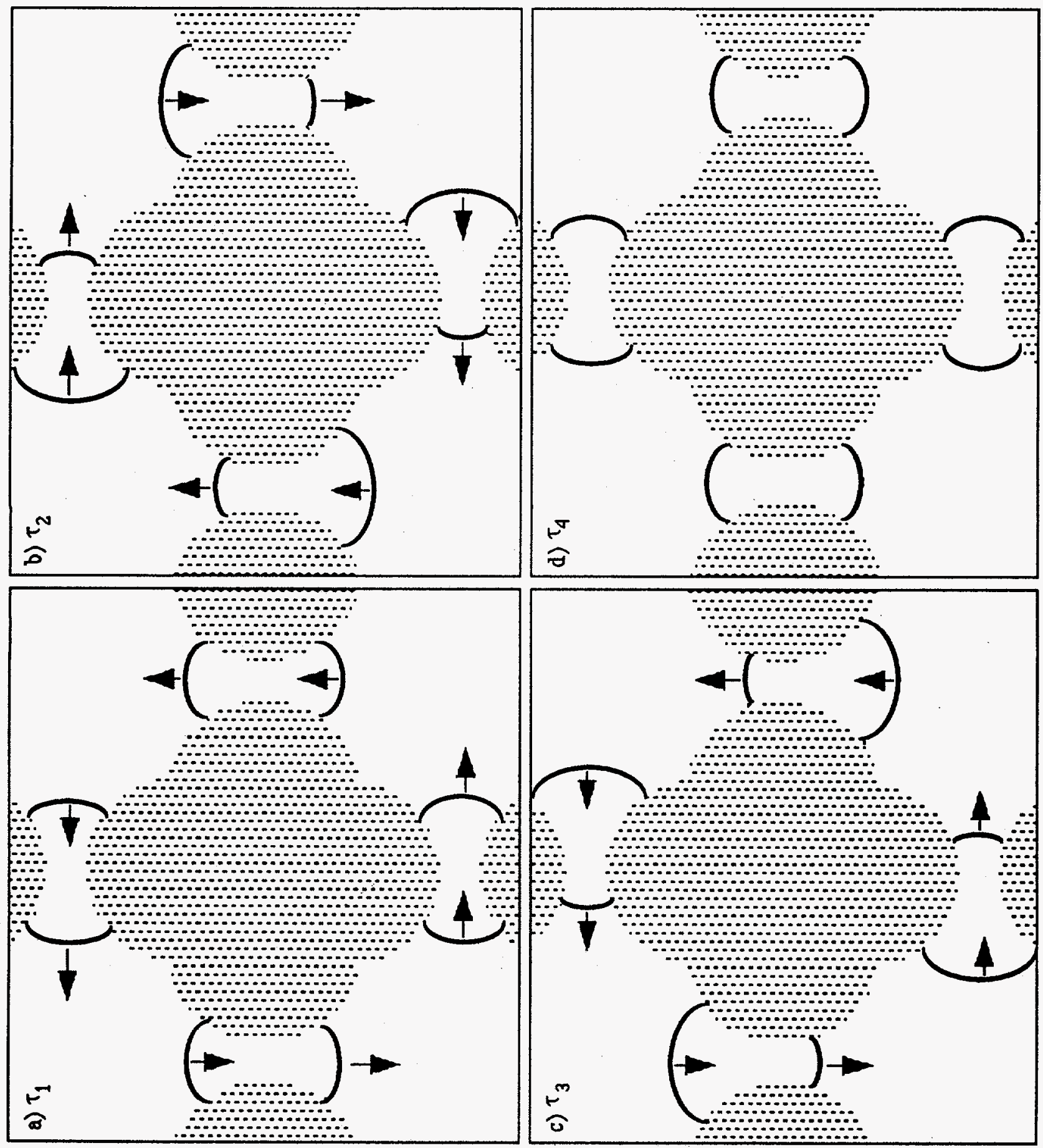


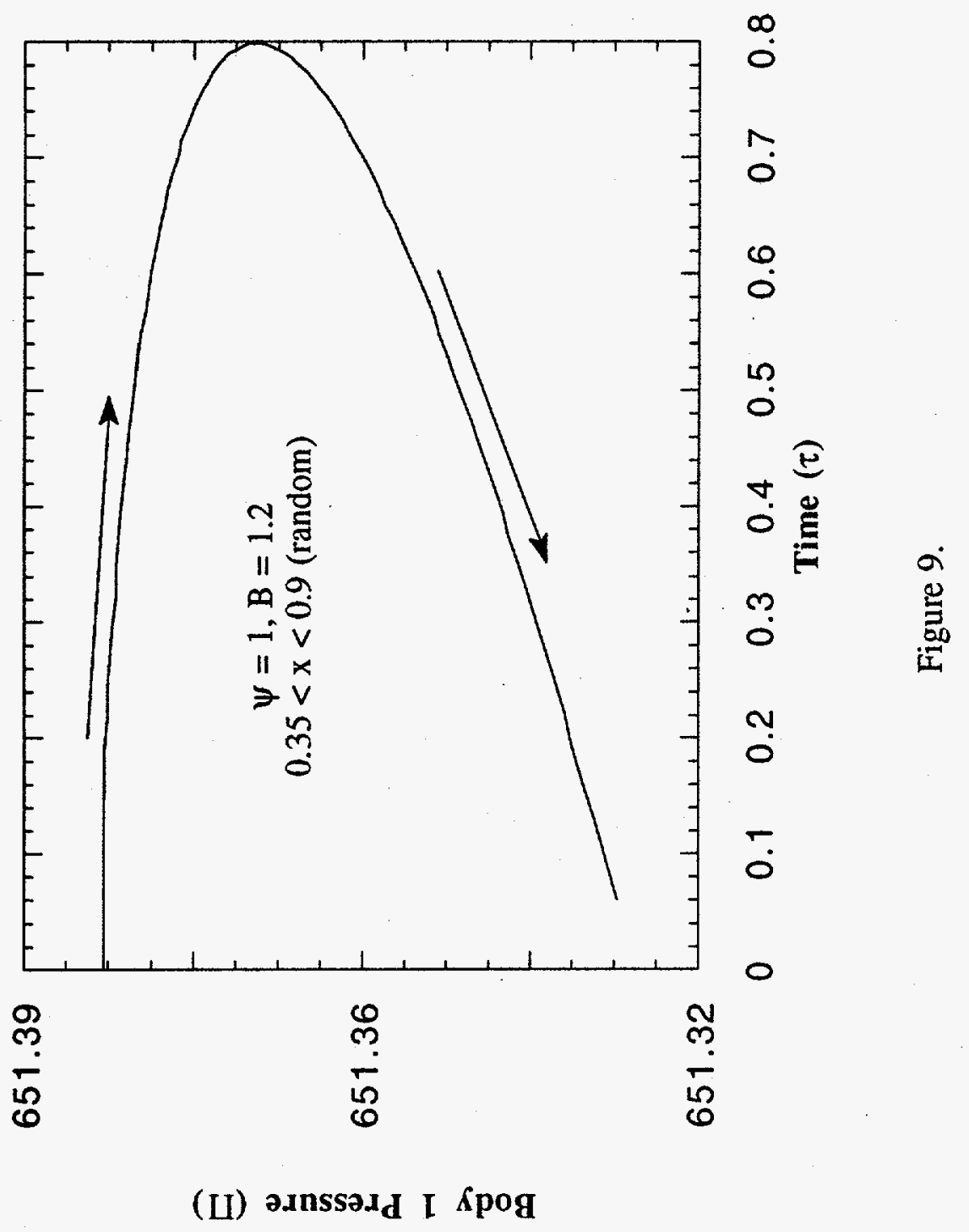




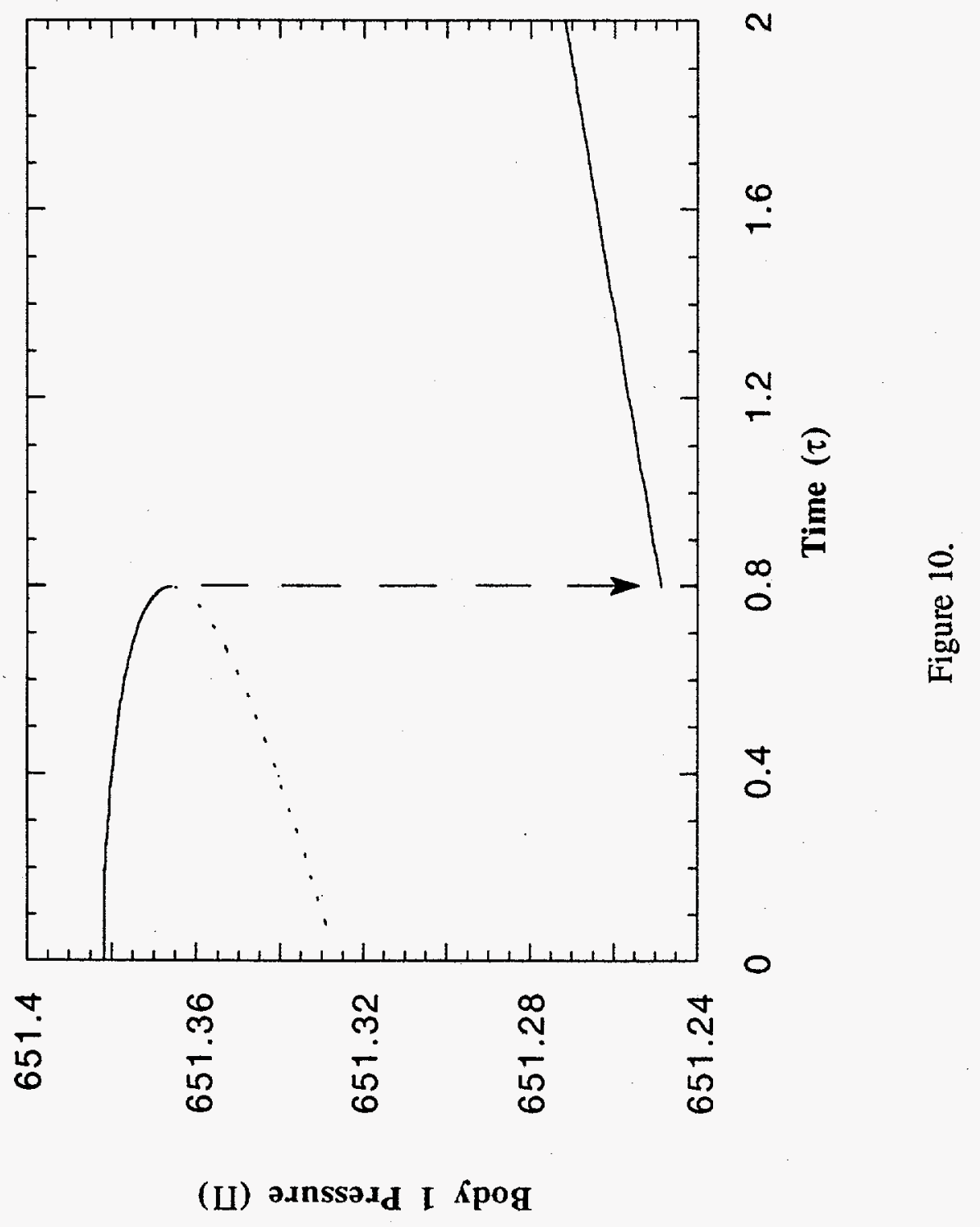




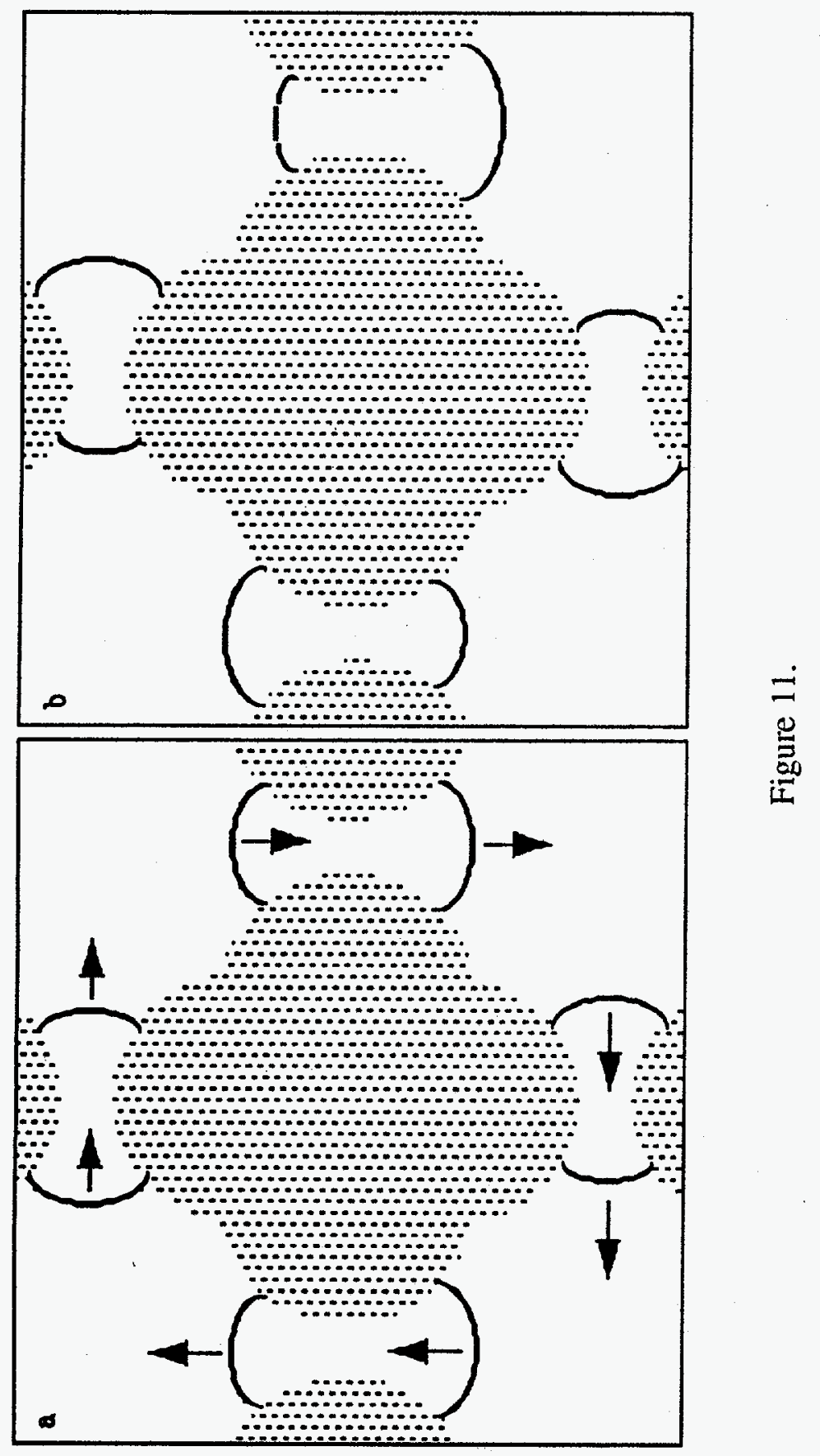



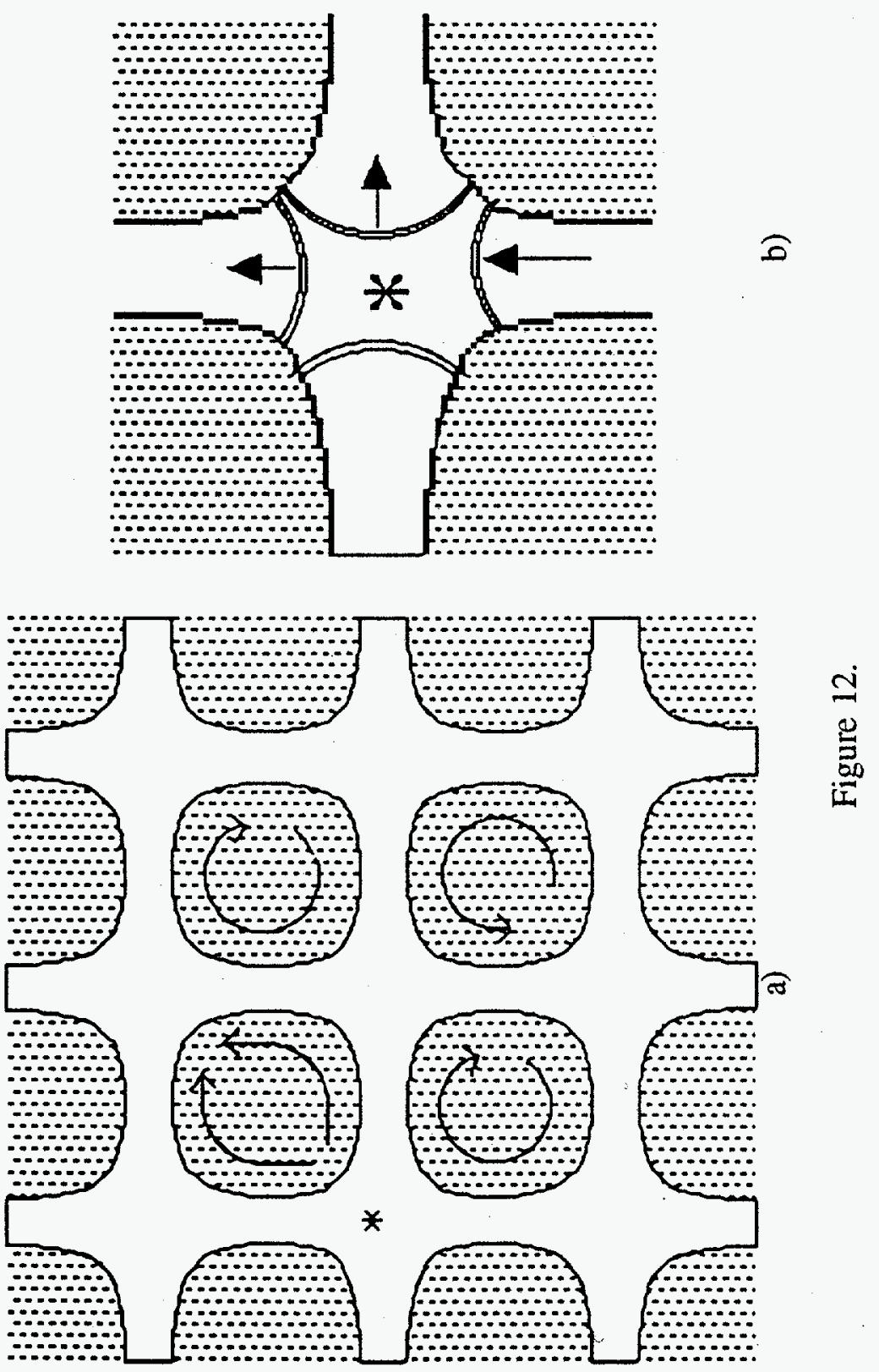


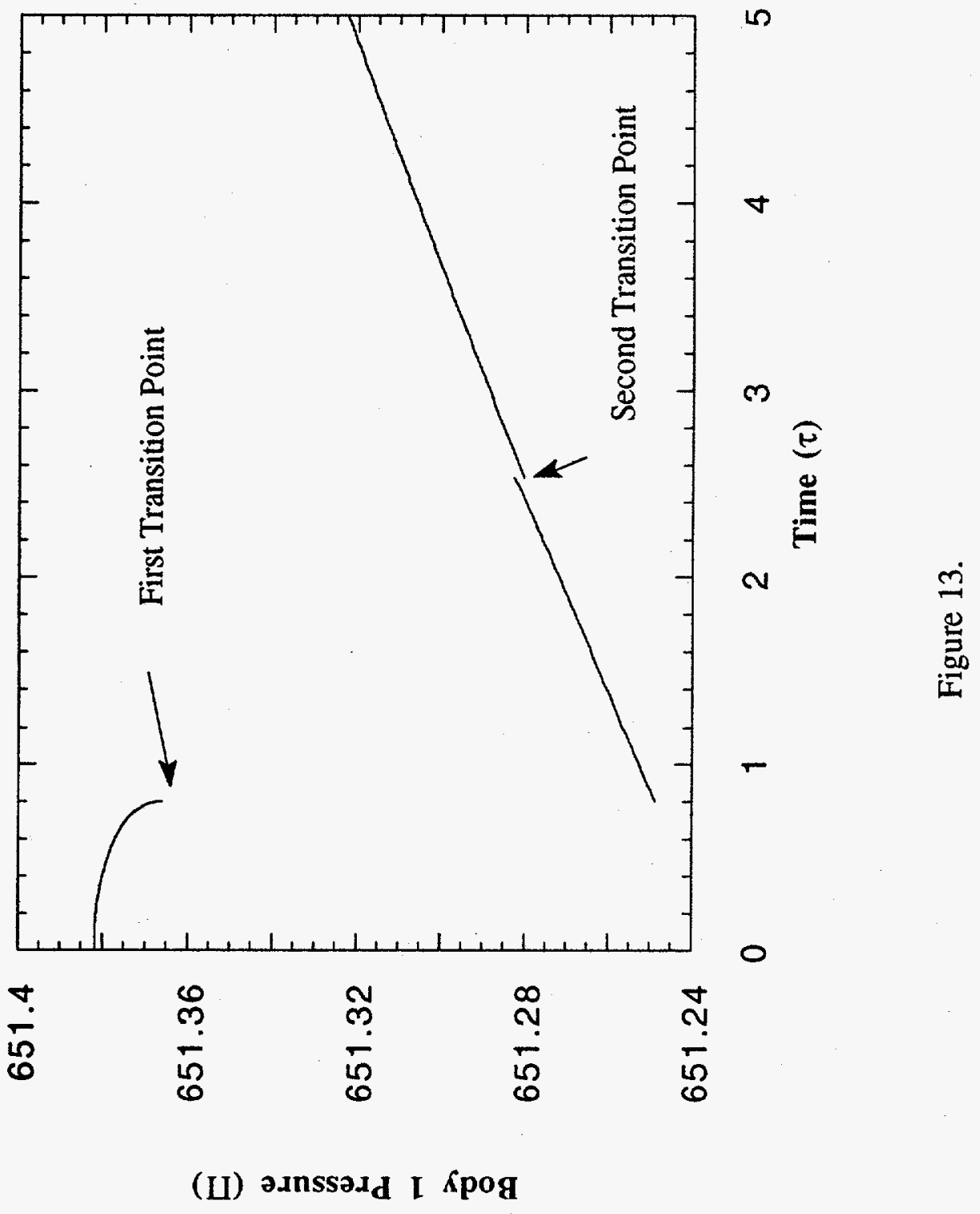


Lamella Position (x)

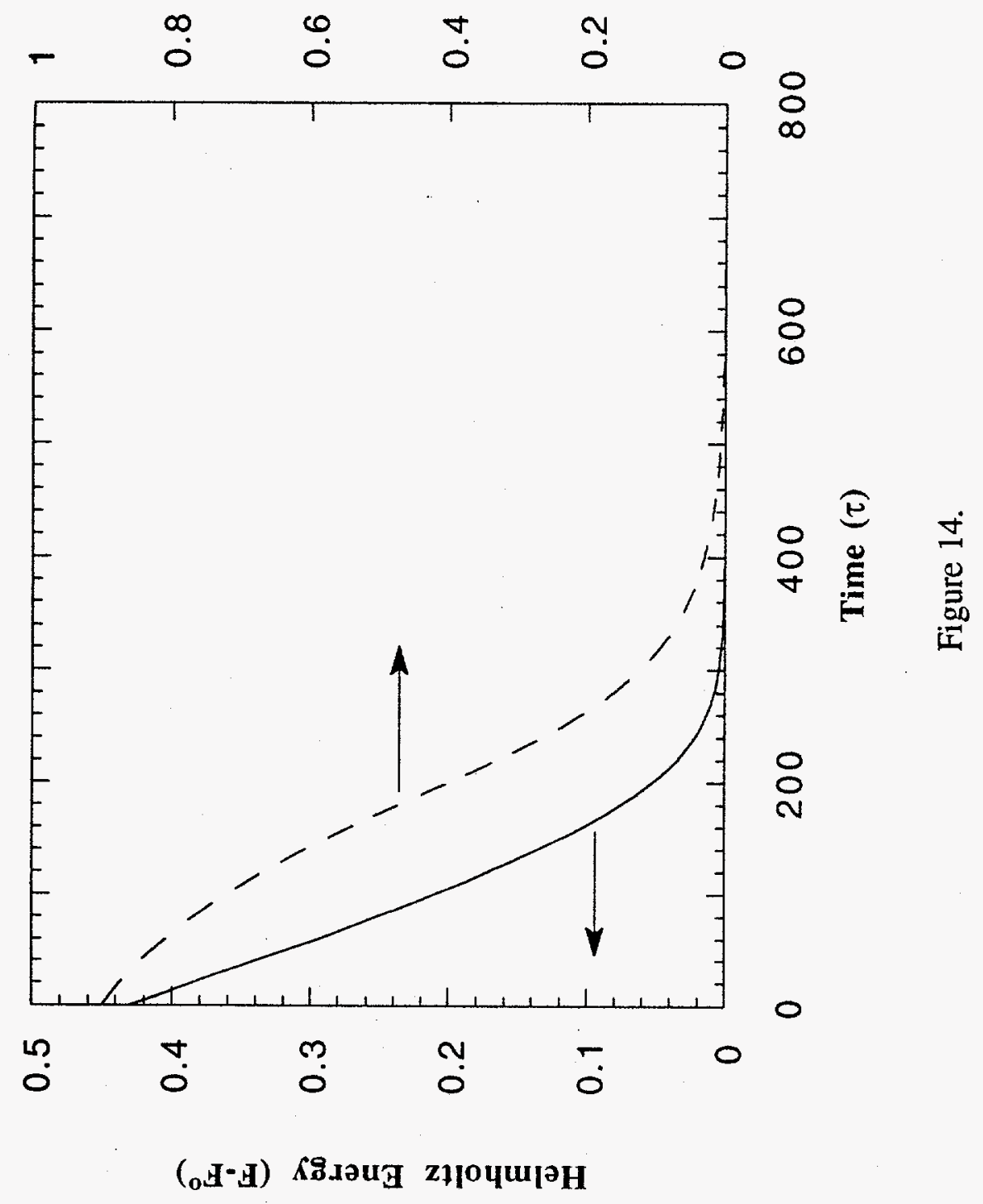




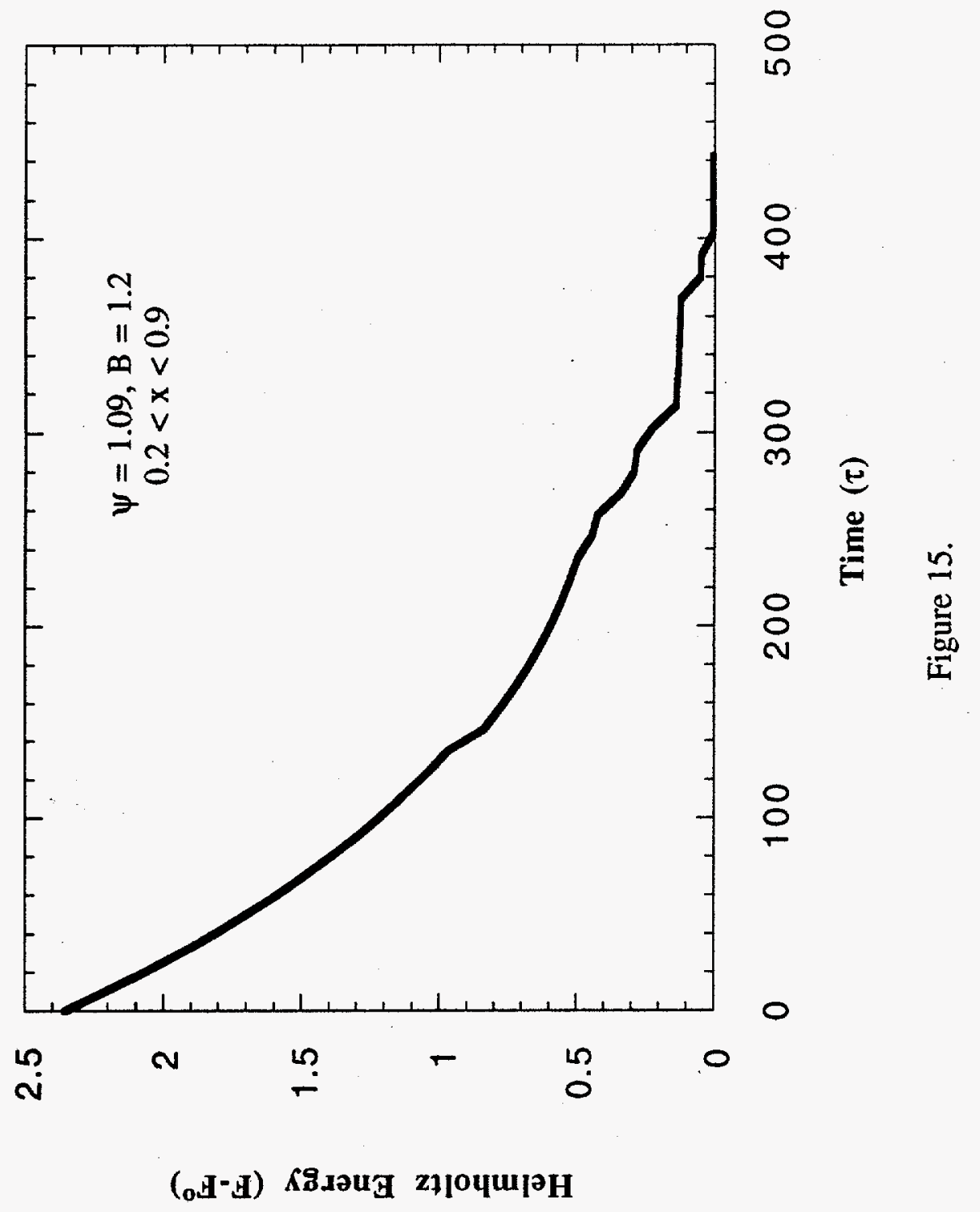




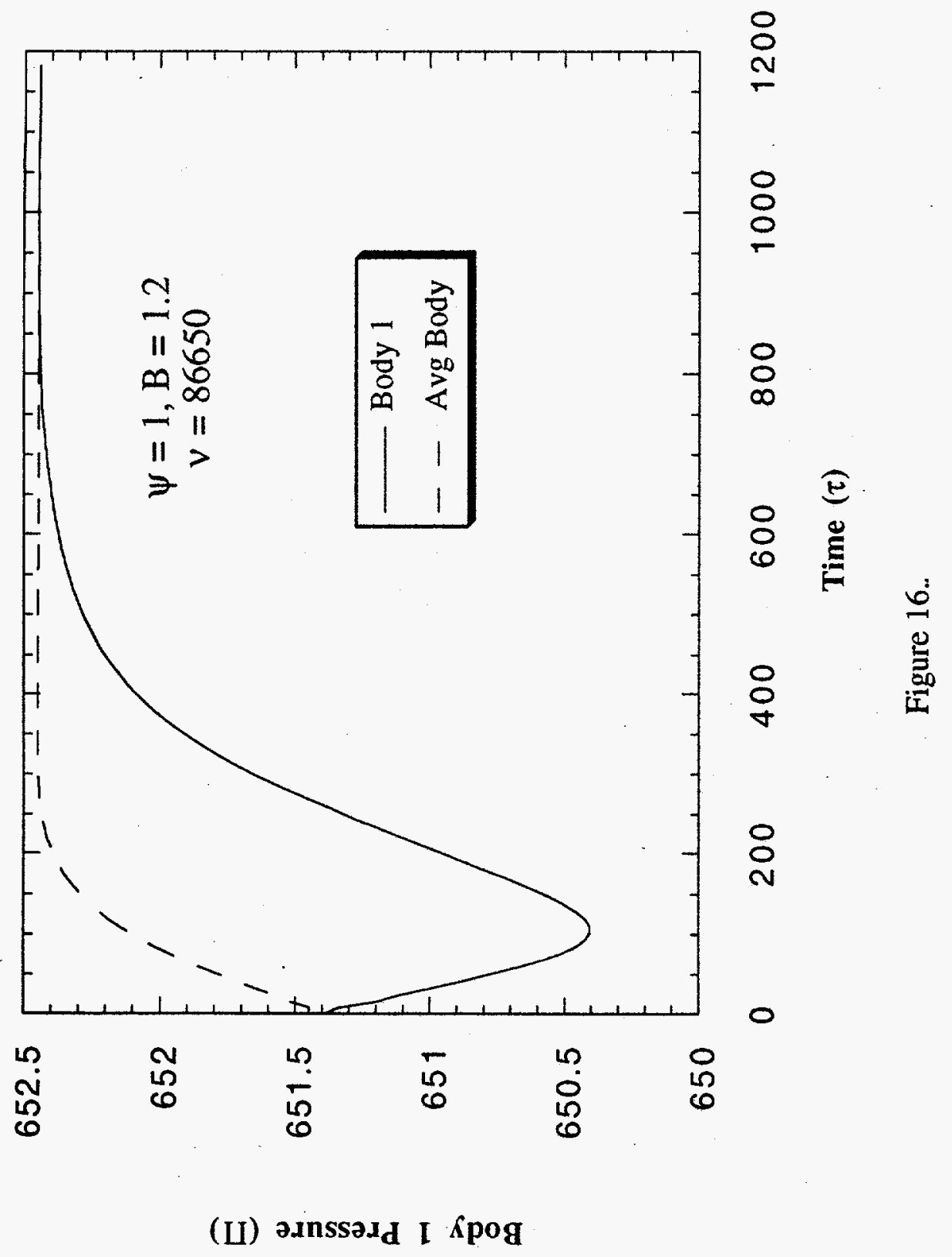




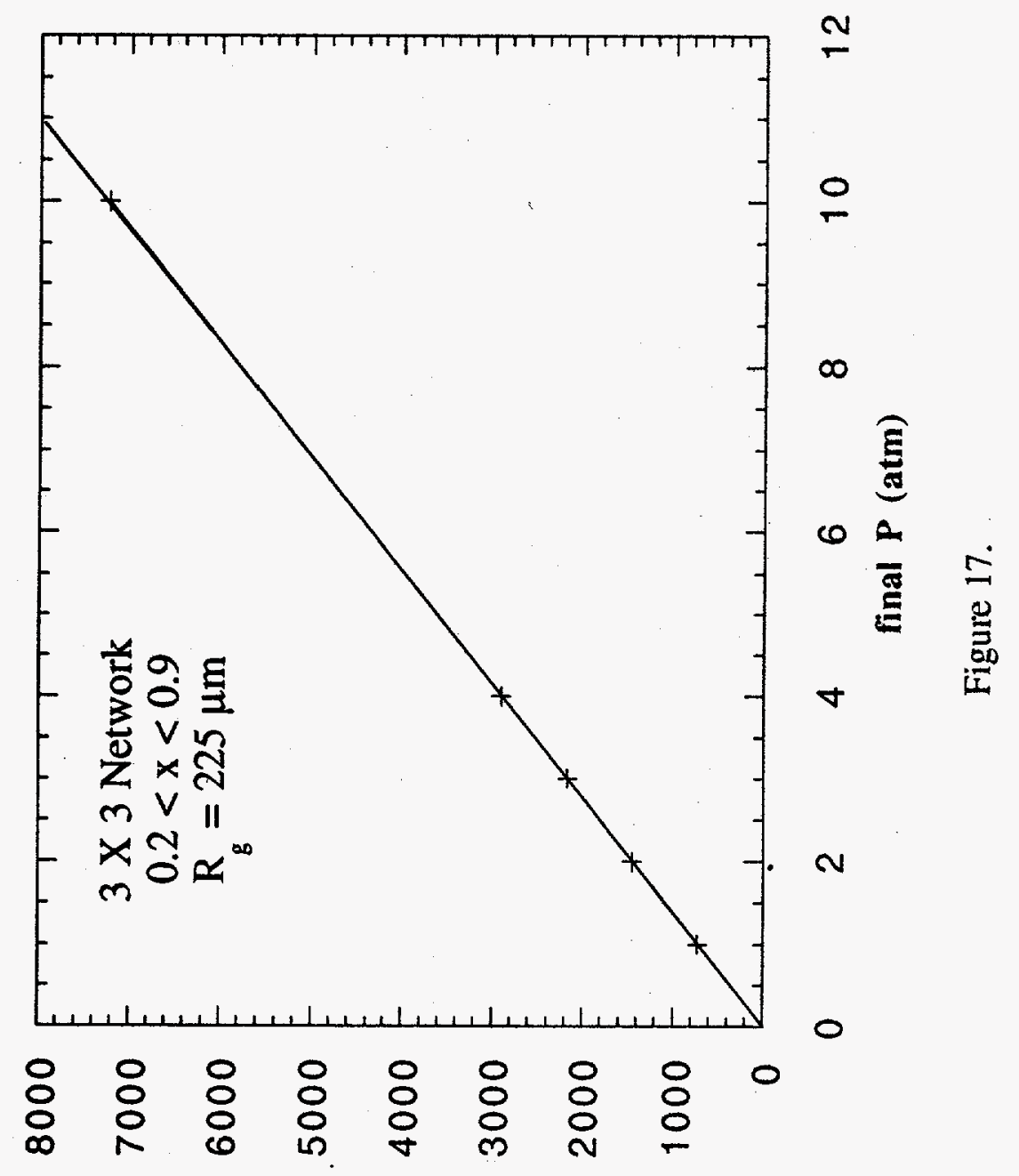

(s) әน!ฺ Кеวәр 


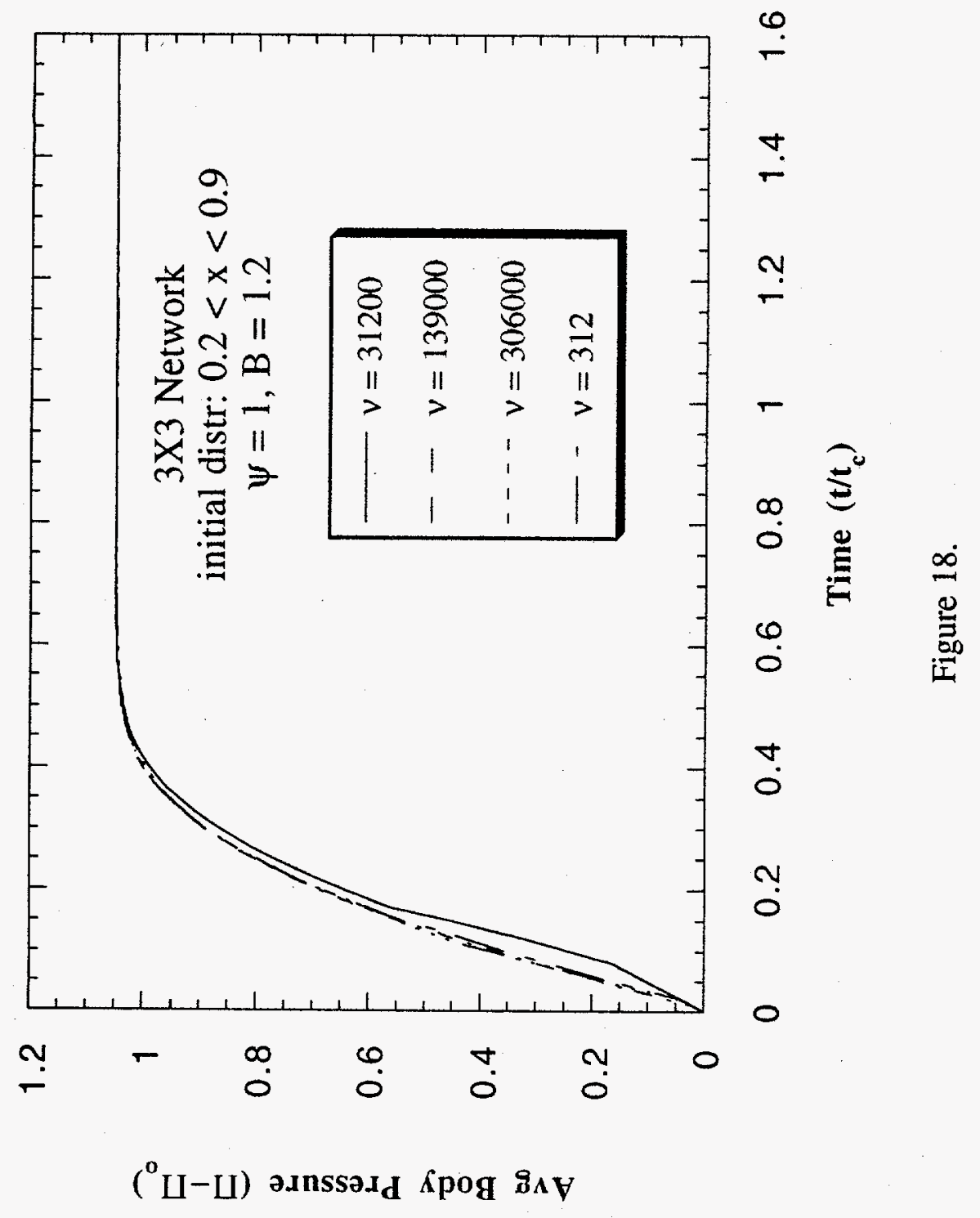




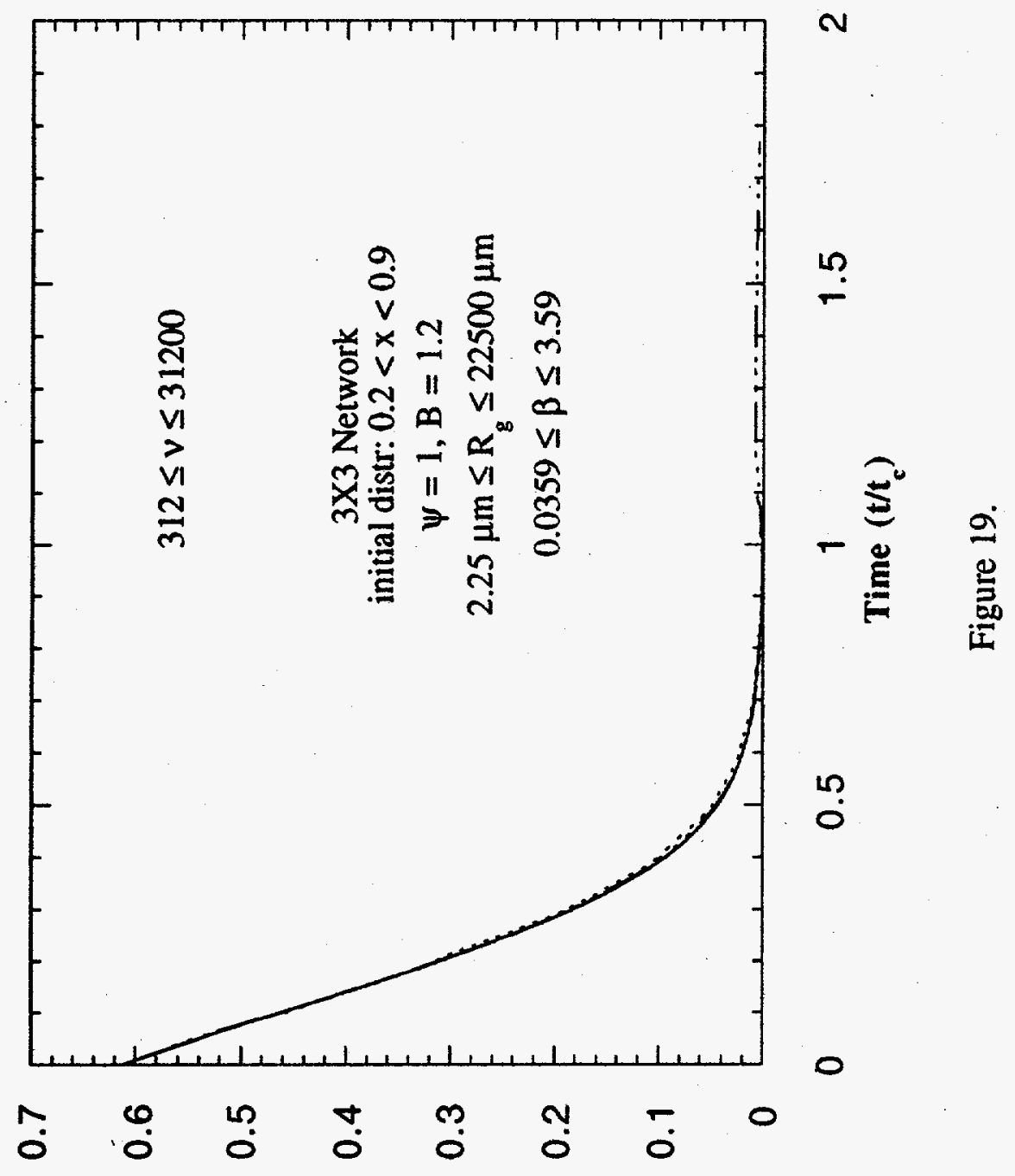

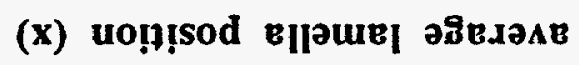




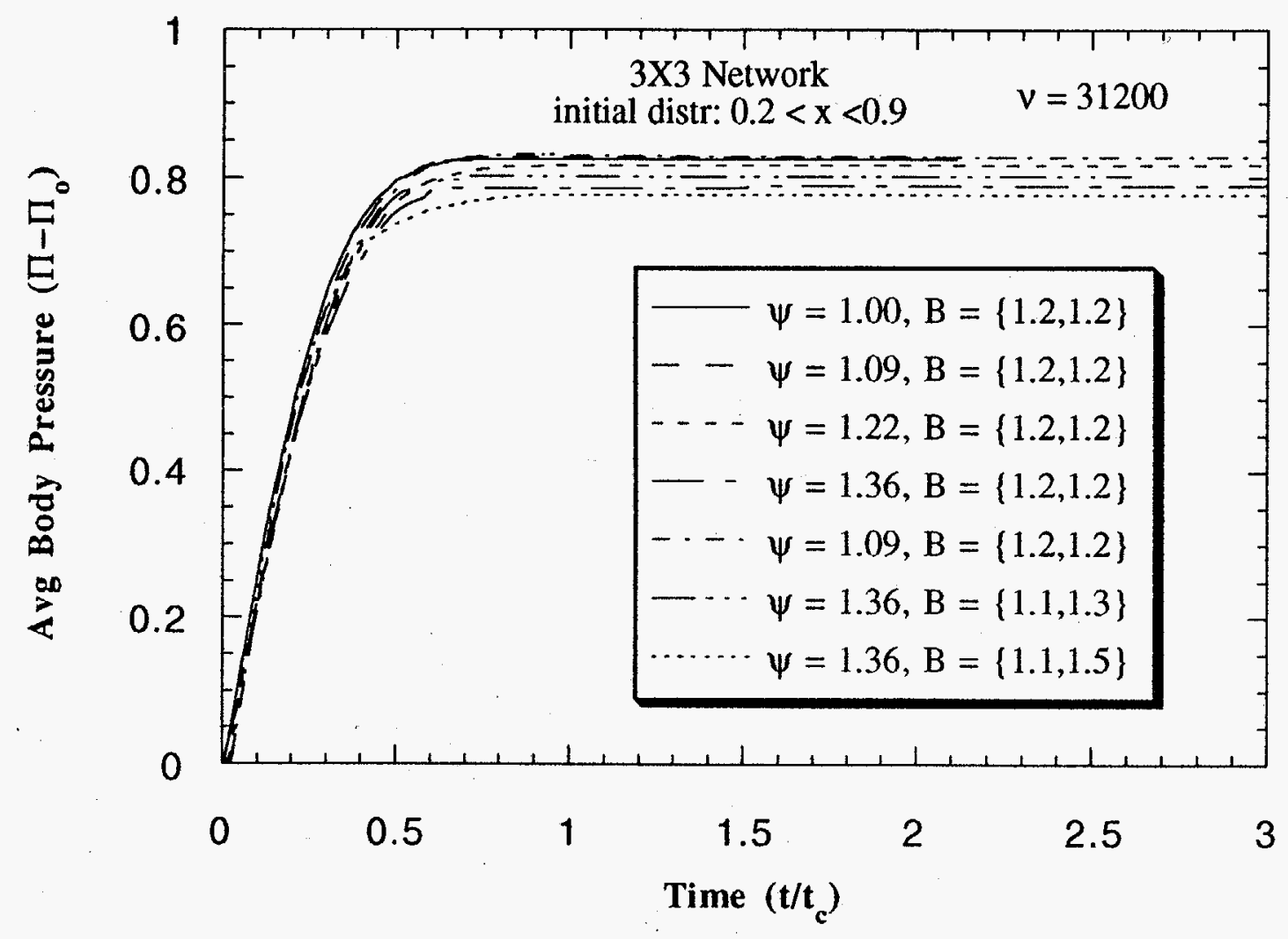

Figure 20. 


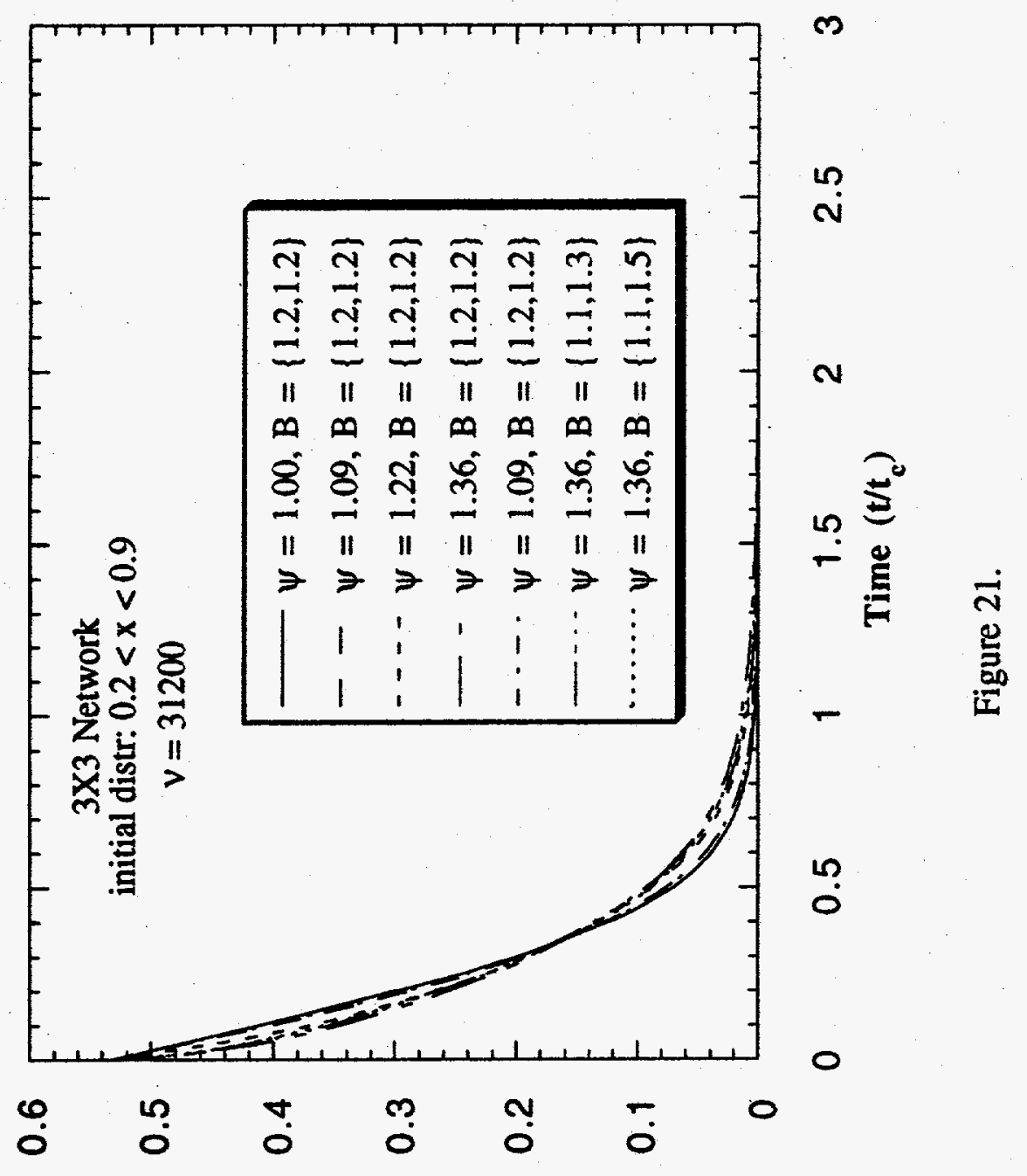

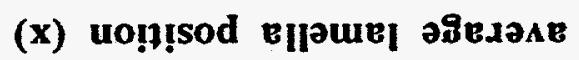

